\title{
Cinnarizine- and flunarizine-associated movement disorder: a literature review
}

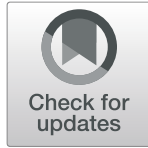

Jamir Pitton Rissardo * (D) and Ana Letícia Fornari Caprara

\begin{abstract}
Introduction: Cinnarizine (CNZ) and flunarizine (FNZ) belong to the calcium channel blockers class of medication.

Main text: The aim of this literature review is to evaluate the clinical epidemiological profile, pathological mechanisms, and management of CNZ/FNZ-associated movement disorder (MD). Relevant reports in six databases were identified and assessed by two reviewers without language restriction. One hundred and seventeen reports containing 1920 individuals who developed a CNZ/FNZ-associated MD were identified. The MD encountered were 1251 parkinsonism, 23 dyskinesias, 11 akathisia, 16 dystonia, and 5 myoclonus, and in the group not clearly defined, 592 extrapyramidal symptoms, 19 tremors, 2 bradykinesia, and 1 myokymia. The predominant sex was female with a percentage of $72.69 \%$ (466/641). The mean age was 74.49 (SD, 7.88) years. The mean CNZ dose was $148.19 \mathrm{mg}$ $(\mathrm{SD}, 42.51)$ and for the FNZ dose, $11.22 \mathrm{mg}$ (5.39). The mean MD onset and recovery were 1.83 years $(\mathrm{SD}, 1.35)$ and 3.71 months (SD, 1.26). In the subgroup of subjects that had improvement of the symptoms, the complete recovery was achieved within 6 months of the drug withdrawal in almost all subjects (99\%). The most common management was drug withdrawal. A complete recovery was observed in $93.77 \%$ of the patients (437/466).

Conclusions: CNZ/FNZ-associated MD was extensively reported in the literature. Parkinsonism was the most well described. Myoclonus (MCL) was the poorest described MD with missing data about the neurological examination and electrodiagnostic studies. The knowledge of this disorder probably can contribute to the understanding of the other drug-induced MDs.
\end{abstract}

Keywords: Cinnarizine, Flunarizine, Review, Movement disorder, Drug-induced

\section{Key messages}

1. PKN is the most common described MD secondary to CNZ/FNZ treatment.

2. CNZ/FNZ mechanism of action is the blockage of dopamine, histamine, serotonin, and intracellular calcium-calmodulin complex.

3. All MD can be explained by the dopaminergic hypothesis, except MCL that is probably associated with serotonin.

* Correspondence: jamirrissardo@gmail.com

Medicine Department, Federal University of Santa Maria, Rua Roraima, Santa Maria, Rio Grande do Sul, Brazil

\section{Springer Open}

\section{Introduction}

Cinnarizine (CNZ) and flunarizine (FNZ) belong to the calcium channel blockers family (Fig. 1). In this context, CNZ was first synthesized in 1955 by chemistries of the Janssen Pharmaceutica in attempts to develop a new antihistamine drug [1]; animal studies showed that CNZ inhibits vascular smooth muscle contraction, and this action occurs especially in the intracranial vessels [2]. So the idea of a drug with "cerebral vasodilation" properties was evolving [3]; this was probably even more encouraged due to the increasing knowledge about the cerebrovascular diseases [4]. Apparently, a safety profile was observed, and CNZ was marketed in the middle 1960s [1].

FNZ was discovered in the same pharmaceutical company as CNZ in 1961; it was designed to have a better dosage form and increase the effectiveness of its dubious

(c) The Author(s). 2020 Open Access This article is licensed under a Creative Commons Attribution 4.0 International License, which permits use, sharing, adaptation, distribution and reproduction in any medium or format, as long as you give appropriate credit to the original author(s) and the source, provide a link to the Creative Commons licence, and indicate if changes were made. The images or other third party material in this article are included in the article's Creative Commons licence, unless indicated otherwise in a credit line to the material. If material is not included in the article's Creative Commons licence and your intended use is not permitted by statutory regulation or exceeds the permitted use, you will need to obtain permission directly from the copyright holder. To view a copy of this licence, visit http://creativecommons.org/licenses/by/4.0/. 


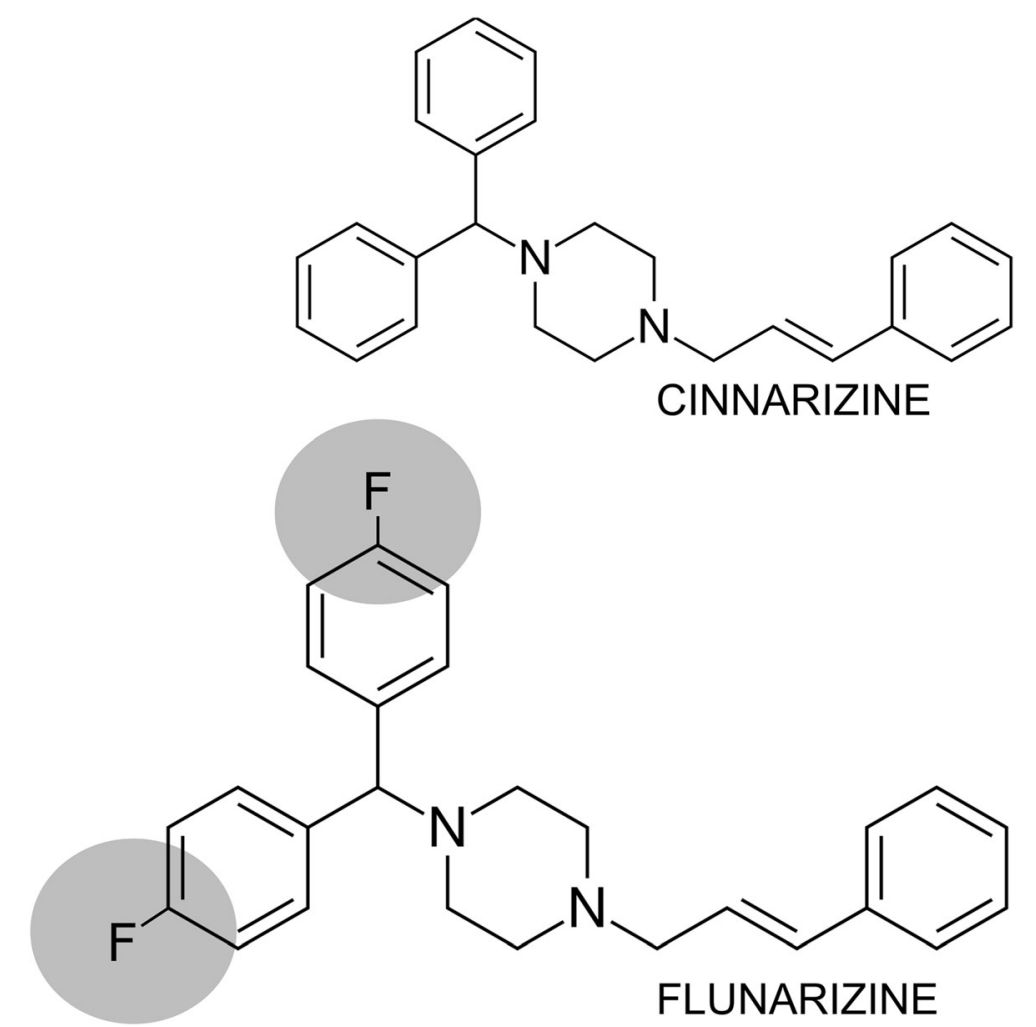

Fig. 1 Skeletal formula of cinnarizine and flunarizine

predecessor [5]. In animal studies, FNZ showed an efficacy of 2.5 to 15 times stronger than CNZ [6]. It was first marketed in Europe around the 1980s about 10 years after the release of CNZ [3]. Spain among the European countries was one of the first in which FNZ was available [6] and probably the country with the greatest number of CNZ/FNZ prescriptions; in 1985, approximately $5 \%$ of the Spanish population over sixty were on CNZ treatment [3].

CNZ/FNZ has been used for several conditions including central and peripheral vascular disorders and balance disorders. Migraine, Raynaud syndrome, Ménière's disease, vertigo, and tinnitus are examples of common indications of these drugs. The mechanism of action of CNZ/FNZ is poorly understood (Fig. 2) [2, 6-11]. These medications were first described as calcium channel blockers with activity in the L/T-type channels [6], but more recent studies showed that the main action of CNZ/FNZ may not be inhibiting calcium entry into cells, but rather by an intracellular mechanism such as antagonism of calmodulin $[2,11]$. It is worthy of mentioning that this mechanism is postulated to be effective for the treatment of vertigo [2]. Other theorized interactions of this drug include the blockage of H1 histamine, 5-HT2c serotonin, and D2 dopamine receptors $[6,10]$. The serotoninergic mechanism is still poorly understood, some studies showed an increase [7] and in others a decrease [10] of the serotonin concentration in the synaptic cleft [6]. Thereby, we believe that these contradictory results probably occurred because different parts of the brain were studied.

The side effects observed in the first clinical studies of this drug in more than $1 \%$ of the population were drowsiness, nausea, indigestion, weight gain, feeling tired, stomachache, vomiting, sweating, and skin rashes [6]. Other adverse events were only reported in the postmarketing experience [12]. Some patients showed symptoms similar to Parkinson's disease such as bradykinesia and resting tremors [13]. Later, many reports of druginduced parkinsonism and other abnormal movements were reported with CNZ/FNZ [13-15], which sometimes are difficult to diagnosis in the clinical practice due to preexisting neurological and psychiatric comorbidities. In this way, the aim of this literature review is to evaluate the clinical epidemiological profile, pathological mechanisms, and management of CNZ- and FNZassociated movement disorders.

\section{Methods}

Search strategy

We searched six databases in an attempt to locate any and all existing reports on movement disorders (MD) 


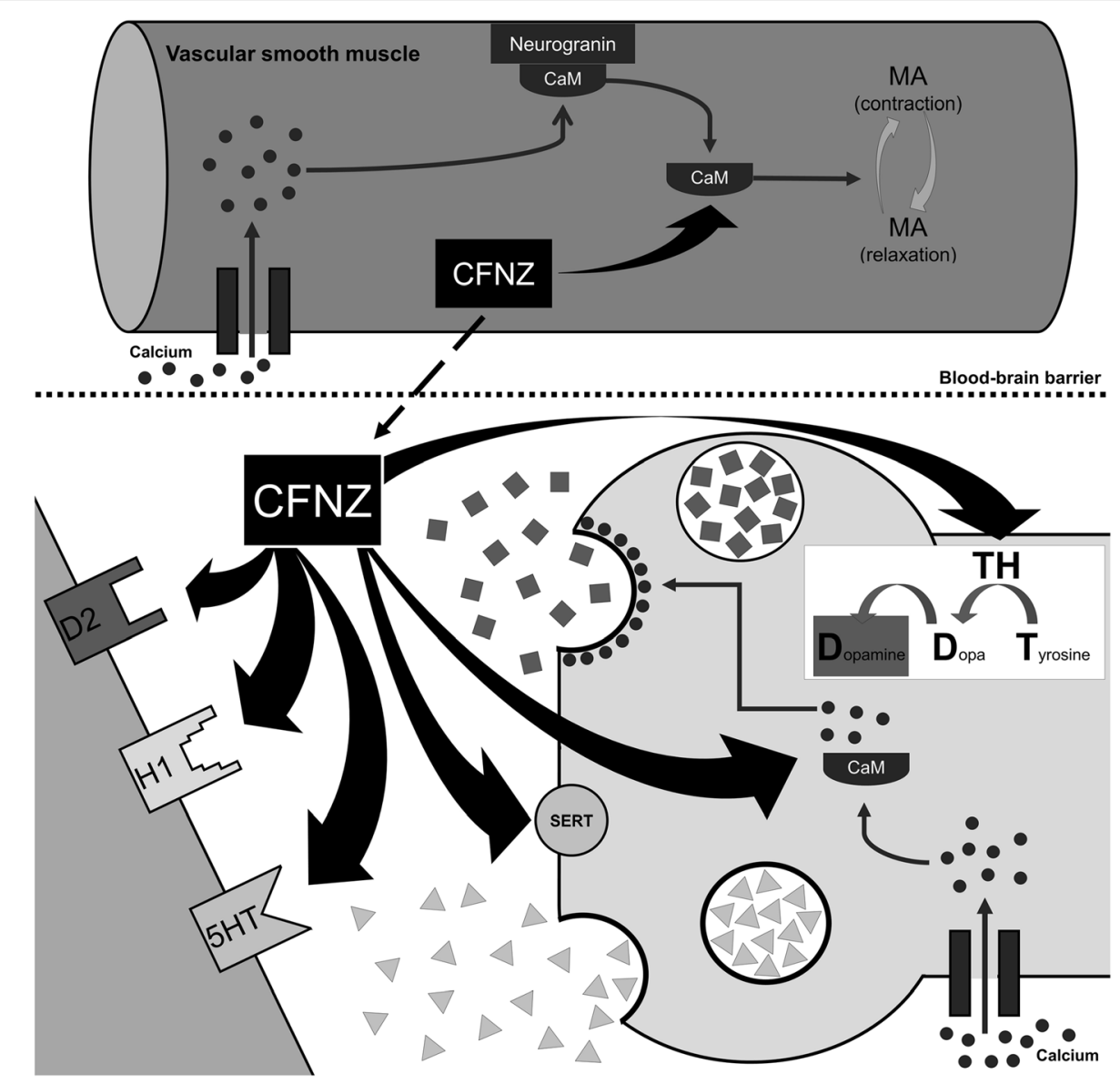

Fig. 2 Schematic diagram of the mechanism of action of cinnarizine and flunarizine (CFNZ). CFNZ can act in the vascular smooth muscle blocking the complex calcium-calmodulin (CaM), which will inhibit muscle contraction. In the central nervous system, CFNZ has six main actions; blockage of dopamine D2, histamine $\mathrm{H1}$, and serotonin receptors. Also, it can inhibit the tyrosine hydroxylase and CaM decreasing the synthesis and release of dopamine. Moreover, depending on the site of action, CFNZ can increase serotonin concentration inhibiting serotonin reuptake (SERT) and facilitating serotonin release

secondary to CNZ and FNZ published between 1980 and 2019 in electronic form. Excerpta Medica (Embase), Google Scholar, Latin American and Caribbean Health Sciences Literature (Lilacs), MEDLINE, Scientific Electronic Library Online (Scielo), and ScienceDirect were searched. Search terms were "parkinsonism, dyskinesia, dystonia, stuttering, myoclonus, restless legs syndrome, akathisia, tremor, chorea, tics, restlessness, ataxia, ballism, hyperkinetic, hypokinetic, bradykinesia, movement disorder." These terms were combined with "cinnarizine, flunarizine" (Other 1 - Supplementary material).

\section{Inclusion and exclusion criteria}

Case reports, case series, original articles, letters to the editor, bulletins, and poster presentations published from 1980 to 2019 were included in this review with no language restriction. The authors independently screened the titles and abstracts of all papers found from the initial search. Disagreements between the authors were resolved through discussion.

Cases where the cause of MD was already known and either motor symptoms did not worsen or were not related to CNZ/FNZ were excluded. Also, cases that were not accessible by electronic methods including after a formal request to the authors of the study by email were excluded. Cases that had more than one factor contributing to the MD were evaluated based on the probability of the event occurrence based on the Naranjo algorithm.

\section{Data extraction}

For CNZ/FNZ, a total of 4032 papers were found; 3431 were irrelevant and 484 were unrelated to the complication, duplicate, inaccessible electronically, or provided insufficient data (Fig. 3). Data abstraction was done. When provided, we extracted author, department, year of publication, country of origin, number of patients affected, CNZ/FNZ indication including off-label uses, 


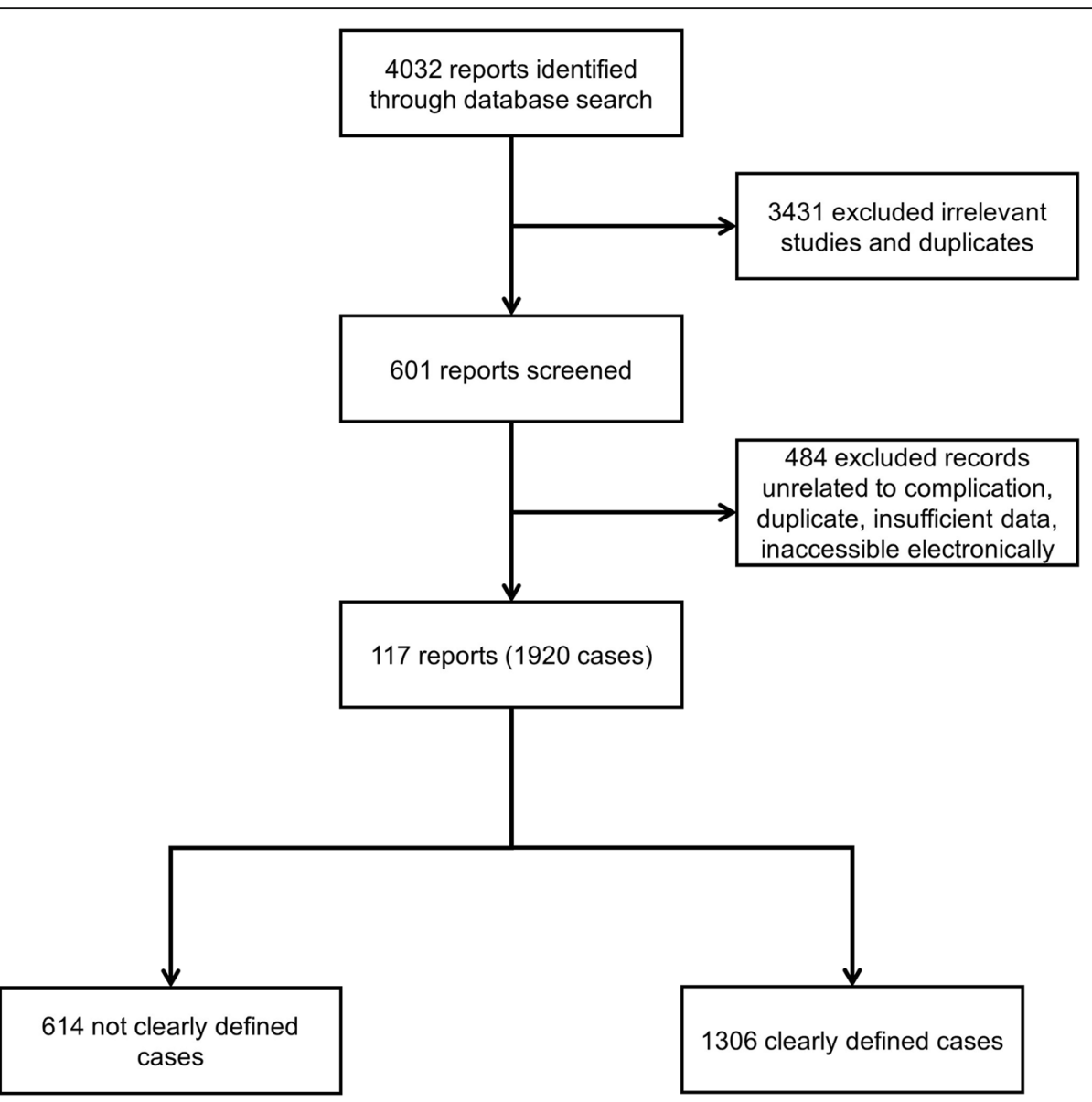

Fig. 3 Flow chart of the screening process for cinnarizine and flunarizine

time from first CNZ/FNZ dose till MD onset, time from CNZ/FNZ withdrawal to symptoms improvement, patient's status at follow-up, and important findings of clinical history and management. The majority of the reports did not provide sufficient information about the times of MD onset and recovery. The data were extracted by two independent authors, double-checked to ensure matching, and organized by whether the MD was a side effect of the CNZ/FNZ use.

\section{Statistical analysis}

Categorical variables were represented as proportions; continuous variables were represented as mean, standard deviation (SD), median, and range.

\section{Definitions}

The clinical characteristics and definitions of the MDs such as parkinsonism, tics, dyskinesia, dystonia, myoclonus, restless legs syndrome, akathisia, tremor, chorea, ataxia, and ballism were obtained from the reference Jankovic and Tolosa [16]. The clinical diagnosis for the psychiatric conditions was obtained from the diagnostic and statistical manual of mental disorders (DSM- $5^{\circ}$ ) [17]. The Naranjo algorithm was used for determining the likelihood of whether an adverse drug reaction was actually due to the drug rather than the result of other factors [18]. In the cases where the non-English literature was beyond the authors' proficiency (English, Portuguese, Spanish, Italian, French, and German) and the English abstract did not provide enough data such as Japanese, Korean, Chinese, Russian, and Dutch, Google Translate service was used [19].

\section{Results}

For the years 1980 and 2019, a total of 117 reports containing 1920 individuals who developed a movement disorder associated with CNZ/FNZ were identified from 27 countries (Table 1) [3-5, 12-15, 20-129]. The origin was Asian in 834, European 663, South America 415, and North America 8. Figure 4 shows the number of reports associated with movement disorders and $\mathrm{CNZ}$ / FNZ over time with important markers of the history of the CNZ/FNZ-induced parkinsonism [13, 15, 53, 73, 79, $87,115,130]$. The movement disorders encountered 
Table 1 Clinical reports of CNZ/FNZ-associated MD

\begin{tabular}{|c|c|c|c|c|c|c|c|c|c|c|}
\hline Reference & $\begin{array}{l}\text { Country/ } \\
\text { year }\end{array}$ & $\begin{array}{l}N \\
\text { cases }\end{array}$ & Age & Sex & $\begin{array}{l}\text { Suspected } \\
\text { drug }\end{array}$ & $\begin{array}{l}\text { Drug } \\
\text { dose } \\
\text { (mg) }\end{array}$ & $\begin{array}{l}\text { Time from } \\
\text { drug-start } \\
\text { to } \\
\text { symptoms }\end{array}$ & $\begin{array}{l}\text { Time from } \\
\text { withdrawal } \\
\text { to recovery }\end{array}$ & $\begin{array}{l}\text { Follow- } \\
\text { up }\end{array}$ & $\begin{array}{l}\text { Important clinical history and } \\
\text { clinical management }\end{array}$ \\
\hline \multicolumn{11}{|l|}{ Parkinsonism } \\
\hline Mello-Souza & Brazil 1984 & 5 & NA & NA & FNZ & NA & NA & NA & NA & \\
\hline $\begin{array}{l}\text { Martí-Masso } \\
\text { et al. }\end{array}$ & Spain 1985 & 11 & $65-83$ & NA & CNZ & 150 & $\begin{array}{l}6-36 \\
\text { months }\end{array}$ & NA & NA & \\
\hline \multirow[t]{12}{*}{ Chouza et al. } & \multirow[t]{12}{*}{$\begin{array}{l}\text { Uruguay } \\
1986\end{array}$} & \multirow[t]{12}{*}{12} & 70 & F & FNZ & $\begin{array}{l}10-20 \\
m g\end{array}$ & 9 months & NA & No & $\begin{array}{l}\text { CH: PKN +orofacial DKN } \\
\text { +severe AKT +moderate DPS. } \\
\text { Even after } 20 \text { months of drug } \\
\text { withdrawal, she was still with } \\
\text { severe AKT. CM: Drug } \\
\text { withdrawal }\end{array}$ \\
\hline & & & 70 & $\mathrm{~F}$ & FNZ & 20 & 6 months & NR & $C R$ & $\begin{array}{l}\text { CH: PKN +mild DPS. CM: Drug } \\
\text { withdrawal }\end{array}$ \\
\hline & & & 45 & $\mathrm{~F}$ & FNZ & $20-40$ & 2 months & NR & $C R$ & $\mathrm{CH}: \mathrm{PKN}$ + moderate DPS \\
\hline & & & 56 & M & FNZ & 20 & 1 month & NR & $C R$ & $\begin{array}{l}\text { CH: PKN +mild DPS. CM: Drug } \\
\text { withdrawal }\end{array}$ \\
\hline & & & 66 & $\mathrm{~F}$ & FNZ & $20-40$ & $\begin{array}{l}\text { Several } \\
\text { months }\end{array}$ & NR & $C R$ & $\begin{array}{l}\text { CH: PKN +severe DPS. CM: } \\
\text { Drug withdrawal }\end{array}$ \\
\hline & & & 63 & M & FNZ & 20 & $\begin{array}{l}13 \\
\text { months }\end{array}$ & NR & $C R$ & $\begin{array}{l}\text { CH: PKN +rabbit syndrome } \\
\text { (DKN) +mild DPS. CM: Drug } \\
\text { withdrawal }\end{array}$ \\
\hline & & & 55 & $\mathrm{~F}$ & FNZ & 10 & $\begin{array}{l}15 \\
\text { months }\end{array}$ & NR & $C R$ & $\begin{array}{l}\mathrm{CH}: \text { PKN + moderate DPS. CM: } \\
\text { Drug withdrawal }\end{array}$ \\
\hline & & & 71 & $\mathrm{~F}$ & FNZ & 20 & $\begin{array}{l}12 \\
\text { months }\end{array}$ & NR & $C R$ & $\begin{array}{l}\text { CH: PKN +orofacial DKN +mild } \\
\text { DPS. CM: Drug withdrawal }\end{array}$ \\
\hline & & & 72 & $\mathrm{~F}$ & FNZ & 40 & 1 month & NR & $C R$ & $\begin{array}{l}\text { CH: PKN +mild AKT +moderate } \\
\text { DPS. CM: Drug withdrawal }\end{array}$ \\
\hline & & & 65 & M & FNZ & 10 & NR & NR & $C R$ & $\begin{array}{l}\mathrm{CH} \text { : PKN +moderate DPS. CM: } \\
\text { Drug withdrawal }\end{array}$ \\
\hline & & & 66 & $\mathrm{~F}$ & FNZ & 40 & 1 month & $N R$ & $C R$ & $\begin{array}{l}\text { CH: PKN +rabbit syndrome } \\
\text { (DKN) +moderate DPS. CM: } \\
\text { Drug withdrawal }\end{array}$ \\
\hline & & & 73 & $\mathrm{~F}$ & FNZ & 20 & 3 months & NR & $C R$ & $\begin{array}{l}\text { CH: Only PKN. CM: Drug } \\
\text { withdrawal }\end{array}$ \\
\hline \multirow[t]{6}{*}{$\begin{array}{l}\text { D'Alessandro } \\
\text { et al. }\end{array}$} & \multirow[t]{6}{*}{ Italy 1986} & \multirow[t]{6}{*}{6} & 67 & $\mathrm{~F}$ & FNZ & 10 & NR & 6 months & $C R$ & $\begin{array}{l}\text { CH: PKN +DPS. CM: Drug } \\
\text { withdrawal }\end{array}$ \\
\hline & & & 72 & M & FNZ & 20 & NR & 3 months & $C R$ & $\begin{array}{l}\text { CH: PKN +DPS. CM: Drug } \\
\text { withdrawal and amitriptyline } \\
\text { started }\end{array}$ \\
\hline & & & 72 & M & FNZ & 10 & NR & NA & No & $\begin{array}{l}\text { CH: PKN +DPS. CM: Drug } \\
\text { withdrawal and amineptine } \\
\text { started }\end{array}$ \\
\hline & & & 77 & $\mathrm{~F}$ & FNZ & 20 & NR & 3 months & $C R$ & $\begin{array}{l}\text { CH: PKN +DPS. CM: Drug } \\
\text { withdrawal and amitriptyline } \\
\text { started }\end{array}$ \\
\hline & & & 77 & $F$ & FNZ & 10 & NR & 3 months & $C R$ & $\begin{array}{l}\text { CH: PKN +DPS. CM: Drug } \\
\text { withdrawal and amitriptyline } \\
\text { started }\end{array}$ \\
\hline & & & 82 & M & FNZ & 10 & $N R$ & NA & No & $\begin{array}{l}\text { CH: PKN +DPS. CM: Drug } \\
\text { withdrawal }\end{array}$ \\
\hline \multirow{3}{*}{$\begin{array}{l}\text { Laporte and } \\
\text { Capella }\end{array}$} & \multirow[t]{3}{*}{ Spain 1986} & \multirow[t]{3}{*}{14} & 78 & M & CNZ & NR & 4.5 years & $N R$ & NR & \multirow{3}{*}{$\begin{array}{l}\mathrm{CH} \text { : All had some tremor or } \\
\text { bradykinesia; two individuals } \\
\text { had to worsen PD. CM: Drug } \\
\text { withdrawal }\end{array}$} \\
\hline & & & 46 & $\mathrm{~F}$ & CNZ & 45 & 5 days & NR & No & \\
\hline & & & 48 & $\mathrm{~F}$ & CNZ & $N R$ & 14 & $N R$ & $N R$ & \\
\hline
\end{tabular}


Table 1 Clinical reports of CNZ/FNZ-associated MD (Continued)

\begin{tabular}{|c|c|c|c|c|c|c|c|c|c|c|}
\hline Reference & $\begin{array}{l}\text { Country/ } \\
\text { year }\end{array}$ & $\begin{array}{l}N \\
\text { cases }\end{array}$ & Age & Sex & $\begin{array}{l}\text { Suspected } \\
\text { drug }\end{array}$ & $\begin{array}{l}\text { Drug } \\
\text { dose } \\
\text { (mg) }\end{array}$ & $\begin{array}{l}\text { Time from } \\
\text { drug-start } \\
\text { to } \\
\text { symptoms }\end{array}$ & $\begin{array}{l}\text { Time from } \\
\text { withdrawal } \\
\text { to recovery }\end{array}$ & $\begin{array}{l}\text { Follow- } \\
\text { up }\end{array}$ & $\begin{array}{l}\text { Important clinical history and } \\
\text { clinical management }\end{array}$ \\
\hline & & & & & & & months & & & \\
\hline & & & 68 & M & CNZ & 150 & 2 days & NR & $C R$ & \\
\hline & & & 73 & M & CNZ & 150 & NR & NR & $C R$ & \\
\hline & & & 69 & $\mathrm{~F}$ & CNZ & 150 & NR & NR & No & \\
\hline & & & 64 & $\mathrm{~F}$ & CNZ & 60-180 & 1 year & NR & No & \\
\hline & & & 68 & M & CNZ & 150 & 2 days & NR & $C R$ & \\
\hline & & & 64 & $\mathrm{~F}$ & FNZ & 10 & 28 days & NR & $C R$ & \\
\hline & & & 42 & M & FNZ & 10 & 4 months & NR & $C R$ & \\
\hline & & & 48 & $\mathrm{~F}$ & FNZ & 20 & 11 days & NR & No & \\
\hline & & & 60 & $\mathrm{~F}$ & FNZ & 10 & 6 weeks & NR & $C R$ & \\
\hline & & & 68 & M & FNZ & 10 & 1 year & NR & $C R$ & \\
\hline & & & 69 & $\mathrm{~F}$ & FNZ & 10 & 8 months & NR & No & \\
\hline $\begin{array}{l}\text { Meyboom } \\
\text { et al. }\end{array}$ & $\begin{array}{l}\text { Netherlands } \\
1986\end{array}$ & 1 & 68 & M & FNZ & 10 & $N R$ & $\begin{array}{l}\text { Several } \\
\text { months }\end{array}$ & $C R$ & $\begin{array}{l}\text { CH: PKN +AKT +mild DPS. CM: } \\
\text { Drug withdrawal }\end{array}$ \\
\hline $\begin{array}{l}\text { Martí-Masso } \\
\text { et al. }\end{array}$ & Spain 1987 & 4 & 62.5 (mean) & NA & CNZ & 150 & 58 days & NR & NA & $\begin{array}{l}\mathrm{CH} \text { : Randomized trial of CNZ in } \\
10 \text { patients with PD. After } 1 \\
\text { month, } 40 \%(4 / 10) \text { had to } \\
\text { withdraw from the study due } \\
\text { to the worsening of } \\
\text { bradykinesia and tremor }\end{array}$ \\
\hline Michele et al. & Italy 1987 & 10 & $61-77$ & NA & FNZ & NA & NA & NA & NA & $\begin{array}{l}\text { Discusses the range of } \\
\text { symptoms of PKN +DPS } \\
\text { associated with dosages of } \\
\text { FNZ }\end{array}$ \\
\hline \multirow[t]{11}{*}{ Micheli et al. } & \multirow{11}{*}{$\begin{array}{l}\text { Argentina } \\
1987\end{array}$} & \multirow[t]{11}{*}{11} & 69 & M & FNZ & 10 & 1 month & 3 months & $C R$ & CM: Drug withdrawal \\
\hline & & & 68 & $\mathrm{~F}$ & CNZ & 225 & 4 years & 5 months & $C R$ & CM: Drug withdrawal \\
\hline & & & 82 & $\mathrm{~F}$ & FNZ & 11.5 & 1 year & 15 days & $C R$ & $\begin{array}{l}\text { CH: PKN +DPS. CM: Drug } \\
\text { withdrawal }\end{array}$ \\
\hline & & & 73 & $\mathrm{~F}$ & CNZ & 150 & 30 days & 17 days & $C R$ & $\begin{array}{l}\text { CH: PKN +DPS. CM: Drug } \\
\text { withdrawal }\end{array}$ \\
\hline & & & 74 & $\mathrm{~F}$ & CNZ & 150 & 4 years & 20 days & $C R$ & $\begin{array}{l}\text { CH: PKN +DPS. CM: Drug } \\
\text { withdrawal }\end{array}$ \\
\hline & & & 61 & $\mathrm{~F}$ & FNZ & 11.5 & 3 months & 5 months & $C R$ & $\begin{array}{l}\text { CH: PKN +DPS +AKT +orofacial } \\
\text { DKN. CM: Drug withdrawal }\end{array}$ \\
\hline & & & 71 & $\mathrm{~F}$ & FNZ & 10 & 3 months & 1 month & $C R$ & CM: Drug withdrawal \\
\hline & & & 73 & $\mathrm{~F}$ & CNZ & 225 & 3 months & 3 months & $C R$ & CM: Drug withdrawal \\
\hline & & & 74 & M & $\mathrm{FNZ}+\mathrm{CNZ}$ & $10+150$ & $\begin{array}{l}7 \\
\text { months- } \\
1 \text { year }\end{array}$ & 1 month & $C R$ & $\begin{array}{l}\mathrm{CH}: \text { PKN +DPS. CM: Drug } \\
\text { withdrawal }\end{array}$ \\
\hline & & & 82 & $\mathrm{~F}$ & FNZ & 10 & 7 months & 24 days & $C R$ & CM: Drug withdrawal \\
\hline & & & 67 & $\mathrm{~F}$ & FNZ & 10 & $\begin{array}{l}16 \\
\text { months }\end{array}$ & 6 months & $C R$ & $\begin{array}{l}\text { CH: PKN +DPS. CM: Drug } \\
\text { withdrawal }\end{array}$ \\
\hline di Rosa et al. & Italy 1987 & 42 & Elderly & NA & FNZ & NR & Months & 12 weeks & NA & CM: Drug withdrawal \\
\hline $\begin{array}{l}\text { Bakchine } \\
\text { et al. }\end{array}$ & France 1988 & 1 & 68 & $\mathrm{~F}$ & FNZ & 10 & 10 weeks & 3 months & $C R$ & $\begin{array}{l}\text { CH: PKN +AKT +orofacial DKN } \\
\text { +DPS. CM: Drug withdrawal }\end{array}$ \\
\hline $\begin{array}{l}\text { Benvenuti } \\
\text { et al. }\end{array}$ & Italy 1988 & 27 & 74 (mean) & $\begin{array}{l}19 \mathrm{~F} \\
+ \\
8 \mathrm{M}\end{array}$ & FNZ & 10 & $\begin{array}{l}14 \\
\text { months } \\
\text { (mean) }\end{array}$ & $\begin{array}{l}<6 \text { months } \\
(96 \%)\end{array}$ & $C R$ & CM: Drug withdrawal \\
\hline Capella et al. & Spain 1988 & 39 & 78 (mean) & $24 \mathrm{~F}$ & CNZ & 156 & 14.67 & $<6$ months & $C R$ & $\mathrm{CH}: 3$ patients were taking \\
\hline
\end{tabular}


Table 1 Clinical reports of CNZ/FNZ-associated MD (Continued)

\begin{tabular}{|c|c|c|c|c|c|c|c|c|c|c|}
\hline Reference & $\begin{array}{l}\text { Country/ } \\
\text { year }\end{array}$ & $\begin{array}{l}N \\
\text { cases }\end{array}$ & Age & Sex & $\begin{array}{l}\text { Suspected } \\
\text { drug }\end{array}$ & $\begin{array}{l}\text { Drug } \\
\text { dose } \\
\text { (mg) }\end{array}$ & $\begin{array}{l}\text { Time from } \\
\text { drug-start } \\
\text { to } \\
\text { symptoms }\end{array}$ & $\begin{array}{l}\text { Time from } \\
\text { withdrawal } \\
\text { to recovery }\end{array}$ & $\begin{array}{l}\text { Follow- } \\
\text { up }\end{array}$ & $\begin{array}{l}\text { Important clinical history and } \\
\text { clinical management }\end{array}$ \\
\hline & & & & ++ & & (mean) & (mean) & $(90 \%)$ & & $\begin{array}{l}\text { other drugs (sulpiride, } \\
\text { thiethylperazine, } \\
\text { dimenhydrinate, thioridazine); } \\
8 \text { were with a combination } \\
\text { CNZ+FNZ } 10 \text { mg; } 4 \text { patients } \\
\text { had DPS symptoms. CM: Drug } \\
\text { withdrawal }\end{array}$ \\
\hline \multirow[t]{10}{*}{$\begin{array}{l}\text { Lugaresi } \\
\text { et al. }\end{array}$} & Italy 1988 & 10 & 72 & M & FNZ & 10 & $\begin{array}{l}40 \\
\text { months }\end{array}$ & NR & NR & CM: Drug withdrawal \\
\hline & & & 56 & $\mathrm{~F}$ & FNZ & 10 & 5 months & NR & NR & CM: Drug withdrawal \\
\hline & & & 63 & $\mathrm{~F}$ & FNZ & 10 & $\begin{array}{l}\text { Some } \\
\text { months }\end{array}$ & NR & NR & CM: Drug withdrawal \\
\hline & & & 52 & $\mathrm{~F}$ & FNZ & 10 & 5 months & NR & NR & CM: Drug withdrawal \\
\hline & & & 72 & M & FNZ & 10 & $\begin{array}{l}\text { Some } \\
\text { months }\end{array}$ & NR & NR & CM: Drug withdrawal \\
\hline & & & 63 & $\mathrm{~F}$ & FNZ & 10 & 5 months & NR & NR & CM: Drug withdrawal \\
\hline & & & 61 & $\mathrm{~F}$ & FNZ & 20 & 8 months & NR & NR & CM: Drug withdrawal \\
\hline & & & 70 & M & FNZ & 5 & 6 months & NR & NR & CM: Drug withdrawal \\
\hline & & & 73 & $\mathrm{~F}$ & FNZ & 5 & 9 months & NR & NR & CM: Drug withdrawal \\
\hline & & & 93 & M & FNZ & 10 & 4 months & NR & $N R$ & CM: Drug withdrawal \\
\hline de Marco & Italy 1988 & 1 & Yong adult & M & FNZ & NA & NA & NA & NA & \\
\hline $\begin{array}{l}\text { Martinez- } \\
\text { Lage }\end{array}$ & Spain 1988 & 1 & $\begin{array}{l}35.48 \\
\text { (mean) }\end{array}$ & NR & FNZ & 10 & NA & NA & NA & \\
\hline $\begin{array}{l}\text { Moretti and } \\
\text { Lucantoni }\end{array}$ & Italy 1988 & 24 & 71.1 (mean) & $\begin{array}{l}14 \mathrm{~F} \\
+ \\
10 \mathrm{M}\end{array}$ & FNZ & 10 & $\begin{array}{l}4.2 \\
\text { months } \\
\text { (mean) }\end{array}$ & $\begin{array}{l}<4 \text { months } \\
(50 \%)\end{array}$ & NA & $\begin{array}{l}\text { CH: } 10 \text { individuals had PKN } \\
\text { +DPS }\end{array}$ \\
\hline \multirow[t]{8}{*}{ Fontanari } & Brazil 1989 & 8 & 62 & $\mathrm{~F}$ & FNZ & 10 & 6 months & 3 months & $C R$ & CM: Drug withdrawal \\
\hline & & & 65 & $\mathrm{~F}$ & FNZ & 10 & 5 months & 5 months & $C R$ & CM: Drug withdrawal \\
\hline & & & 68 & $\mathrm{~F}$ & FNZ & 10 & 3 months & 4 months & $C R$ & CM: Drug withdrawal \\
\hline & & & 62 & $\mathrm{~F}$ & FNZ & 10 & $\begin{array}{l}24 \\
\text { months }\end{array}$ & NR & NR & $\begin{array}{l}\text { CH: PKN +orofacial DKN. CM: } \\
\text { drug withdrawal }\end{array}$ \\
\hline & & & 63 & $\mathrm{~F}$ & FNZ & 10 & $\begin{array}{l}18 \\
\text { months }\end{array}$ & 3 months & $C R$ & CM: Drug withdrawal \\
\hline & & & 55 & $\mathrm{~F}$ & FNZ & 10 & 4 months & NR & $C R$ & CM: Drug withdrawal \\
\hline & & & 60 & $\mathrm{~F}$ & FNZ & 10 & 3 months & 6 months & $C R$ & CM: Drug withdrawal \\
\hline & & & 63 & F & FNZ & 10 & 6 months & 6 months & $C R$ & CM: Drug withdrawal \\
\hline $\begin{array}{l}\text { Kuzuhara } \\
\text { et al. }\end{array}$ & Japan 1989 & 31 & Adult & $\begin{array}{l}20 \mathrm{~F} \\
+ \\
7 \mathrm{M}\end{array}$ & FNZ & 10 & $\begin{array}{l}6.1 \\
\text { months } \\
\text { (mean) }\end{array}$ & $\begin{array}{l}<6 \text { months } \\
(90 \%)\end{array}$ & $C R$ & $\begin{array}{l}\mathrm{CH} \text { : Attempts with levodopa, } \\
\text { anticholinergic drugs, and } \\
\text { bromocriptine had been } \\
\text { ineffective until FNZ } \\
\text { withdrawal. } 16 \text { individuals had } \\
\text { PKN+DPS and } 5 \text { PKN+AKT }\end{array}$ \\
\hline \multirow[t]{2}{*}{$\begin{array}{l}\text { Mangone } \\
\text { et al. }\end{array}$} & $\begin{array}{l}\text { Argentina } \\
1989\end{array}$ & 21 & 68.5 (mean) & $\begin{array}{l}16 F \\
+5 \\
M\end{array}$ & FNZ/CNR & NR & $\begin{array}{l}15.7 \\
\text { months } \\
\text { (mean) }\end{array}$ & 2.6 months & $C R$ & CM: Drug withdrawal \\
\hline & & 2 & 68.5 (mean) & $2 \mathrm{~F}$ & FNZ/CNR & NR & NA & NA & NA & $\begin{array}{l}\mathrm{CH} \text { : Worsening of PD } \\
\text { symptoms }\end{array}$ \\
\hline Micheli et al. & $\begin{array}{l}\text { Argentina } \\
1989\end{array}$ & 81 & 69.7 (mean) & $\begin{array}{l}69 \mathrm{~F} \\
+ \\
31 \mathrm{M}\end{array}$ & $\begin{array}{l}51 \mathrm{FNZ/} \\
31 \mathrm{CNZ/} \\
8 \mathrm{CNZ}+\mathrm{FNZ}\end{array}$ & $\begin{array}{l}13.4 / \\
154.4 \\
\text { (mean) }\end{array}$ & $\begin{array}{l}32.1 / 14.1 \\
\text { months } \\
\text { (mean) }\end{array}$ & $\begin{array}{l}80.5 / 105 \\
\text { days } \\
\text { (mean) }\end{array}$ & $C R$ & $\begin{array}{l}\text { CH: } 46 \text { individuals had PKN } \\
\text { +DPS. CM: Drug withdrawal }\end{array}$ \\
\hline Mukai et al. & Japan & 1 & Adult & NA & FNZ & NA & NA & NA & NA & $\mathrm{CH}$ : Showed slightly decreased \\
\hline
\end{tabular}


Table 1 Clinical reports of CNZ/FNZ-associated MD (Continued)

\begin{tabular}{lllll}
\hline Reference & $\begin{array}{l}\text { Country/ } \\
\text { year }\end{array}$ & $\begin{array}{l}N \\
\text { cases }\end{array}$ & Age & Sex $\begin{array}{l}\text { Suspected } \\
\text { drug }\end{array}$
\end{tabular}

Drug
dose
$(\mathrm{mg})$
Time from Time from Follow- Important clinical history and drug-start withdrawal up clinical management to to recovery
symptoms

\begin{tabular}{|c|c|c|c|c|c|c|c|c|c|c|}
\hline & & & & & & & & & & $\begin{array}{l}\text { signal intensity of the } \\
\text { putamen on brain MRI }\end{array}$ \\
\hline \multirow[t]{19}{*}{$\begin{array}{l}\text { Sa and } \\
\text { Heinisch }\end{array}$} & Brazil 1989 & 19 & 75 & M & FNZ & 20 & $\begin{array}{l}10 \\
\text { months }\end{array}$ & 30 days & $C R$ & $\begin{array}{l}\text { CM: Previous of FNZ } \\
\text { withdrawal was attempted } \\
\text { anticholinergic and levodopa } \\
\text { without success }\end{array}$ \\
\hline & & & 62 & $\mathrm{~F}$ & FNZ & 20 & 2 months & 4 months & $C R$ & $\begin{array}{l}\text { CM: Previous of FNZ } \\
\text { withdrawal was attempted } \\
\text { anticholinergic and levodopa } \\
\text { without success }\end{array}$ \\
\hline & & & 71 & M & FNZ & 20 & 9 months & 30 days & $C R$ & \\
\hline & & & 76 & $\mathrm{~F}$ & FNZ & 10 & 2 months & 60 days & $C R$ & $\begin{array}{l}\text { CM: Previous of FNZ } \\
\text { withdrawal was attempted } \\
\text { anticholinergic without } \\
\text { success }\end{array}$ \\
\hline & & & 72 & F & FNZ & 10 & 1 year & 6 months & $C R$ & $\begin{array}{l}\text { CM: Previous of FNZ } \\
\text { withdrawal was attempted } \\
\text { levodopa without success }\end{array}$ \\
\hline & & & 65 & $\mathrm{~F}$ & FNZ & 10 & 8 months & 4 months & $C R$ & \\
\hline & & & 37 & $\mathrm{~F}$ & FNZ & 20 & 8 months & 4 months & $C R$ & $\mathrm{CH}: \mathrm{PKN}+\mathrm{DPS}$ \\
\hline & & & 67 & F & FNZ & 10 & 7 months & 3 months & $C R$ & $\begin{array}{l}\text { CM: Previous of FNZ } \\
\text { withdrawal was attempted } \\
\text { anticholinergic without } \\
\text { success }\end{array}$ \\
\hline & & & 64 & $F$ & FNZ & 10 & 5 months & 60 days & $C R$ & $\begin{array}{l}\text { CH: PKN +DPS. CM: Previous of } \\
\text { FNZ withdrawal was } \\
\text { attempted anticholinergic and } \\
\text { imipramine without success }\end{array}$ \\
\hline & & & 54 & $\mathrm{~F}$ & FNZ & 10 & 6 months & 3 months & $C R$ & $\begin{array}{l}\text { CM: Previous of FNZ } \\
\text { withdrawal was attempted } \\
\text { anticholinergic and imipramine } \\
\text { without success }\end{array}$ \\
\hline & & & 69 & $\mathrm{~F}$ & FNZ & 10 & 1 year & 50 days & $C R$ & $\mathrm{CH}: \mathrm{PKN}+\mathrm{DPS}$ \\
\hline & & & 47 & $\mathrm{~F}$ & FNZ & 10 & 15 days & 7 days & $C R$ & $\mathrm{CH}: \mathrm{PKN}+\mathrm{DPS}$ \\
\hline & & & 72 & $\mathrm{~F}$ & FNZ & 10 & $\begin{array}{l}11 \\
\text { months }\end{array}$ & 30 days & $C R$ & CH: PKN +DPS \\
\hline & & & 72 & $\mathrm{~F}$ & FNZ & 10 & $N R$ & 60 days & $C R$ & $\begin{array}{l}\text { CM: Previous of FNZ } \\
\text { withdrawal was attempted } \\
\text { anticholinergic and levodopa } \\
\text { without success }\end{array}$ \\
\hline & & & 68 & F & FNZ & 10 & $N R$ & 60 days & $C R$ & $\begin{array}{l}\text { CM: Previous of FNZ } \\
\text { withdrawal was attempted } \\
\text { anticholinergic without } \\
\text { success }\end{array}$ \\
\hline & & & 76 & F & FNZ & 10 & $\begin{array}{l}11 \\
\text { months }\end{array}$ & 10 months & $C R$ & $\begin{array}{l}\text { CM: Previous of FNZ } \\
\text { withdrawal was attempted } \\
\text { anticholinergic without } \\
\text { success }\end{array}$ \\
\hline & & & 74 & $\mathrm{~F}$ & FNZ & 40 & 5 months & 60 days & $C R$ & $\begin{array}{l}\text { CH: PKN +DPS. CM: Previous of } \\
\text { FNZ withdrawal was } \\
\text { attempted levodopa without } \\
\text { success }\end{array}$ \\
\hline & & & $N R$ & F & FNZ & 20 & 7 days & 20 days & $C R$ & $\mathrm{CH}: \mathrm{PKN}+\mathrm{DPS}$ \\
\hline & & & 66 & $\mathrm{~F}$ & FNZ & 40 & 3 months & 60 days & $C R$ & $\begin{array}{l}\text { CH: PKN +DPS. CM: Previous of } \\
\text { FNZ withdrawal was }\end{array}$ \\
\hline
\end{tabular}


Table 1 Clinical reports of CNZ/FNZ-associated MD (Continued)

\begin{tabular}{llllllll}
\hline Reference & $\begin{array}{l}\text { Country/ } \\
\text { year }\end{array}$ & $\begin{array}{l}N \\
\text { cases }\end{array}$ & Age & Sex Suspected $\begin{array}{l}\text { Drug } \\
\text { dose } \\
(\mathrm{mg})\end{array}$ & $\begin{array}{l}\text { Time from Time from Follow- Important clinical history and } \\
\text { drug-start withdrawal up } \\
\text { to } \\
\text { symptoms }\end{array}$ \\
\end{tabular}

\begin{tabular}{|c|c|c|c|c|c|c|c|c|c|c|}
\hline & & & & & & & & & & $\begin{array}{l}\text { attempted imipramine without } \\
\text { success }\end{array}$ \\
\hline $\begin{array}{l}\text { Trevisol- } \\
\text { Bittencourt }\end{array}$ & Brazil 1990 & 1 & 72 & M & FNZ & 10 & 8 months & 30 days & $C R$ & $\begin{array}{l}\text { CM: Drug withdrawal and } \\
\text { biperiden started }\end{array}$ \\
\hline Fontanari & Brazil 1990 & 1 & Adult & $\mathrm{F}$ & FNZ & NR & NR & NR & No & $\begin{array}{l}\mathrm{CH} \text { : She had PKN due to FNZ; } \\
\text { the drug was removed, and } \\
\text { she started to developing } \\
\text { choreoathetotic DKN. } \\
\text { Anticholinergics and levodopa } \\
\text { did not ameliorate the DKN } \\
\text { symptoms }\end{array}$ \\
\hline $\begin{array}{l}\text { Gimenez- } \\
\text { Roldan and } \\
\text { Mateo }\end{array}$ & Spain 1991 & 24 & 70.6 (mean) & $\begin{array}{l}15 \mathrm{~F} \\
+ \\
9 \mathrm{M}\end{array}$ & CNZ & $\begin{array}{l}181.3 \\
\text { (mean) }\end{array}$ & $\begin{array}{l}4.2 \text { years } \\
\text { (mean) }\end{array}$ & NR & NR & \\
\hline $\begin{array}{l}\text { Garcia-Ruiz } \\
\text { et al. }\end{array}$ & Spain 1992 & 32 & 72.6 (mean) & $\begin{array}{l}26 \mathrm{~F} \\
+ \\
6 \mathrm{M}\end{array}$ & $\begin{array}{l}4 \mathrm{FNZI} \\
27 \mathrm{CNZ} / \\
1 \mathrm{FNZ}+\mathrm{CNZ}\end{array}$ & $\begin{array}{l}8.75 / \\
122.5 \\
\text { (mean) }\end{array}$ & $\begin{array}{l}15 \\
\text { months } \\
\text { (mean) }\end{array}$ & NR & NR & $\begin{array}{l}\mathrm{CH} \text { : Only } 3 \text { patients had a full } \\
\text { recovery. } 44 \% \text { had PKN +DPS. } \\
\text { Patients younger than } 73 \text { years } \\
\text { recovered better than older } \\
\text { individuals }\end{array}$ \\
\hline $\begin{array}{l}\text { Morgante } \\
\text { et al. }\end{array}$ & Italy 1992 & 4 & Adult & NR & FNZ & NR & NR & NR & NR & $\begin{array}{l}\text { CH } 1 \text { individual FNZ +alpha } \\
\text { methyldopa; } 3 \text { only FNZ }\end{array}$ \\
\hline $\begin{array}{l}\text { Negrotti } \\
\text { et al. }\end{array}$ & Italy 1992 & 25 & Adult & NR & FNZ/CNZ & NR & NR & NR & NR & $\begin{array}{l}\mathrm{CH} \text { : In the CNZ/FNZ-induced } \\
\text { PKN there was a positive } \\
\text { family history for PD or } \\
\text { essential tremor with a greater } \\
\text { percentage than the general } \\
\text { population }\end{array}$ \\
\hline $\begin{array}{l}\text { Amancio } \\
\text { et al. }\end{array}$ & Brazil 1993 & 1 & Adult & NR & FNZ & NR & NR & NR & NR & \\
\hline Cunha et al. & Brazil 1993 & 11 & 67 (mean) & $\begin{array}{l}8 \mathrm{~F} \\
+ \\
3 \mathrm{M}\end{array}$ & FNZ/CNZ & $\begin{array}{l}\text { 20/150 } \\
\text { (mean) }\end{array}$ & $\begin{array}{l}24 \\
\text { months } \\
\text { (mean) }\end{array}$ & $\begin{array}{l}2 \text { months } \\
\text { (mean) }\end{array}$ & $C R$ & CH: 63\% had PKN +DPS. \\
\hline $\begin{array}{l}\text { Galhardo } \\
\text { et al. }\end{array}$ & Brazil 1993 & 1 & 48 & $\mathrm{~F}$ & FNZ & 10 & 3 months & 90 days & $C R$ & $\begin{array}{l}\text { CM: FNZ withdrawal; } \\
\text { methixene and levodopa } \\
\text { started }\end{array}$ \\
\hline Llau et al. & France 1994 & 16 & 65 (mean) & $\begin{array}{l}10 \mathrm{~F} \\
+ \\
6 \mathrm{M}\end{array}$ & FNZ/CNZ & NR & $\begin{array}{l}15.76 \\
\text { months } \\
\text { (mean) }\end{array}$ & NA & NA & \\
\hline $\begin{array}{l}\text { Anjaneyulu } \\
\text { and } \\
\text { Mohandas }\end{array}$ & India 1995 & 2 & NA & NA & FNZ & NA & NA & NA & NA & \\
\hline $\begin{array}{l}\text { Baquero } \\
\text { et al. }\end{array}$ & Spain 1995 & 18 & 66 (mean) & NA & FNZ/CNZ & NA & $\begin{array}{l}1 \text { year } \\
\text { (mean) }\end{array}$ & NA & NA & \\
\hline Claps & Chile 1995 & $>1$ & NA & NA & FNZ & NA & NA & NA & NA & \\
\hline $\begin{array}{l}\text { Handforth } \\
\text { et al. }\end{array}$ & USA 1995 & 1 & 37 & $\mathrm{~F}$ & FNZ & 60 & NA & NA & NA & $\begin{array}{l}\text { CH: Assessment of FNZ for the } \\
\text { treatment of epilepsy in the } \\
\text { USA }\end{array}$ \\
\hline Biary et al. & Arabia 1995 & 1 & 52 & M & FNZ & 10 & $\begin{array}{l}18 \\
\text { months }\end{array}$ & NR & NR & \\
\hline $\begin{array}{l}\text { Jimenez- } \\
\text { Jimenez et al. }\end{array}$ & Spain 1996 & 30 & 70 (mean) & $\begin{array}{l}24 \mathrm{~F} \\
+ \\
6 \mathrm{M}\end{array}$ & FNZ/CNZ & NR & $\begin{array}{l}60.9 \\
\text { months } \\
\text { (mean) }\end{array}$ & $\begin{array}{l}4.5 \text { months } \\
\text { (mean) }\end{array}$ & $C R$ & \\
\hline Lee and Lee & Korea 1996 & 3 & $\begin{array}{l}64.33 \\
\text { (mean) }\end{array}$ & $\begin{array}{l}2 \mathrm{~F} \\
+ \\
1 \mathrm{M}\end{array}$ & FNZ & 10 & $\begin{array}{l}3 \text { months } \\
\text { (mean) }\end{array}$ & 4 months & $C R$ & $\begin{array}{l}\text { CH: } 2 \text { PKN +DPS; } 1 \text { only PKN. } \\
\text { Only one had a full recovery; } \\
\text { others needed to take } \\
\text { levodopa after the event }\end{array}$ \\
\hline
\end{tabular}


Table 1 Clinical reports of CNZ/FNZ-associated MD (Continued)

\begin{tabular}{|c|c|c|c|c|c|c|c|c|c|c|}
\hline Reference & $\begin{array}{l}\text { Country/ } \\
\text { year }\end{array}$ & $\begin{array}{l}N \\
\text { cases }\end{array}$ & Age & Sex & $\begin{array}{l}\text { Suspected } \\
\text { drug }\end{array}$ & $\begin{array}{l}\text { Drug } \\
\text { dose } \\
\text { (mg) }\end{array}$ & $\begin{array}{l}\text { Time from } \\
\text { drug-start } \\
\text { to } \\
\text { symptoms }\end{array}$ & $\begin{array}{l}\text { Time from } \\
\text { withdrawal } \\
\text { to recovery }\end{array}$ & $\begin{array}{l}\text { Follow- } \\
\text { up }\end{array}$ & $\begin{array}{l}\text { Important clinical history and } \\
\text { clinical management }\end{array}$ \\
\hline Martinez & Chile 1996 & $>1$ & NA & NA & FNZ/CNZ & NA & NA & NA & NA & \\
\hline \multirow{4}{*}{$\begin{array}{l}\text { Morgante } \\
\text { et al. }\end{array}$} & \multirow[t]{4}{*}{ Italy 1996} & \multirow[t]{4}{*}{4} & 74 & M & FNZ & 10 & NR & NR & $C R$ & CM: Drug withdrawal \\
\hline & & & 72 & M & FNZ & 20 & NR & NR & $C R$ & CM: Drug withdrawal \\
\hline & & & 93 & $\mathrm{~F}$ & FNZ & 10 & NR & NR & No & CM: Drug withdrawal \\
\hline & & & 62 & M & FNZ & 10 & NR & NR & NR & CM: Drug withdrawal \\
\hline $\begin{array}{l}\text { Negrotti and } \\
\text { Calzeti }\end{array}$ & Italy 1997 & 11 & 69.5 (mean) & $\mathrm{F}$ & $\begin{array}{l}8 \mathrm{FNZI} \\
3 \mathrm{CNZ}\end{array}$ & $10 / 150$ & 7 months & NA & No & $\begin{array}{l}\text { CH: } 6 \text { orofacial DKN; } 3 \text { limb } \\
\text { DKN. No recovery. CM: Drug } \\
\text { withdrawal }\end{array}$ \\
\hline $\begin{array}{l}\text { Cardoso } \\
\text { et al. }\end{array}$ & Brazil 1998 & 20 & NR & NR & $\begin{array}{l}8 \mathrm{FNZI} \\
12 \mathrm{CNZ}\end{array}$ & NR & NR & NR & NR & \\
\hline $\begin{array}{l}\text { Errea-Abad } \\
\text { et al. }\end{array}$ & Spain 1998 & 19 & Elderly & $\begin{array}{l}14 \mathrm{~F} \\
+ \\
5 \mathrm{M}\end{array}$ & $\begin{array}{l}4 \mathrm{FNZI} \\
15 \mathrm{CNZ}\end{array}$ & NR & NR & NR & NR & \\
\hline $\begin{array}{l}\text { Garcia-Ruiz } \\
\text { et al. }\end{array}$ & Spain 1998 & 36 & 71.7 (mean) & $\begin{array}{l}30 \mathrm{~F} \\
+ \\
6 \mathrm{M}\end{array}$ & $\begin{array}{l}\text { 6FNZ/ } \\
29 C N Z / \\
1 F N Z+C N Z\end{array}$ & NR & NR & NR & NR & $\begin{array}{l}\text { CH: Only } 4 \text { individuals had a } \\
\text { full recovery.CM: Drug } \\
\text { withdrawal }\end{array}$ \\
\hline $\begin{array}{l}\text { Marti-Masso } \\
\text { and Poza }\end{array}$ & Spain 1998 & 87 & 75 (mean) & NR & $\begin{array}{l}\text { 13FNZ/ } \\
69 \mathrm{CNZ/} \\
\text { 5FNZ+CNZ }\end{array}$ & & $\begin{array}{l}33 \\
\text { months } \\
\text { (mean) }\end{array}$ & $\begin{array}{l}5 \text { months } \\
\text { (mean) }\end{array}$ & $\begin{array}{l}\text { CR } \\
(90 \%)\end{array}$ & \\
\hline \multirow[t]{4}{*}{$\begin{array}{l}\text { Orti-Pareja } \\
\text { et al. }\end{array}$} & \multirow[t]{4}{*}{ Spain 1999} & 7 & 75.6 (mean) & $\begin{array}{l}5 \mathrm{~F} \\
+ \\
2 \mathrm{M}\end{array}$ & $7 \mathrm{CNZ}$ & NR & $\begin{array}{l}45.8 \\
\text { months }\end{array}$ & NR & NA & $\mathrm{CH}:$ PKN +orofacial DKN \\
\hline & & 1 & 75.6 (mean) & $\mathrm{F}$ & $1 \mathrm{CNZ}$ & NR & NA & NR & NA & $\mathrm{CH}:$ PKN +DTN \\
\hline & & 3 & 75.6 (mean) & $3 F$ & $3 C N Z$ & NR & NA & NR & NA & $\mathrm{CH}: \mathrm{PKN}+\mathrm{AKT}$ \\
\hline & & 3 & 75.6 (mean) & $3 F$ & $3 C N Z$ & NR & NA & NR & NA & $\begin{array}{l}\text { CH: Only PKN. CM: Drug } \\
\text { withdrawal }\end{array}$ \\
\hline $\begin{array}{l}\text { Stucchi- } \\
\text { Portocarrero } \\
\text { et al. }\end{array}$ & Peru 1999 & 1 & 25 & $\mathrm{~F}$ & CNZ & NA & 11 days & NA & NA & $\begin{array}{l}\text { CH: PKN +AKT +DPS. CM: Drug } \\
\text { withdrawal; benzodiazepines, } \\
\text { propranolol, and orphenadrine } \\
\text { were started }\end{array}$ \\
\hline \multirow[t]{9}{*}{$\begin{array}{l}\text { Zamora and } \\
\text { Argote }\end{array}$} & \multirow[t]{9}{*}{$\begin{array}{l}\text { Colombia } \\
1999\end{array}$} & \multirow[t]{9}{*}{9} & 65 & $\mathrm{~F}$ & FNZ & 10 & 4 years & NA & NA & $\begin{array}{l}\mathrm{CH} \text { : Possible interaction with } \\
\text { verapamil }\end{array}$ \\
\hline & & & 77 & $\mathrm{~F}$ & FNZ & 10 & 1 year & NA & NA & $\begin{array}{l}\mathrm{CH} \text { : Possible interaction with } \\
\text { verapamil }\end{array}$ \\
\hline & & & 65 & M & FNZ & 10 & 6 months & NA & NA & \\
\hline & & & 76 & M & FNZ & 10 & 6 months & NA & NA & \\
\hline & & & 51 & $\mathrm{~F}$ & FNZ & 10 & 3 months & NA & NA & $\mathrm{CH}: \mathrm{PKN}+\mathrm{DPS}$ \\
\hline & & & 51 & $\mathrm{~F}$ & FNZ & 10 & NR & NA & NA & \\
\hline & & & 57 & $\mathrm{~F}$ & CNZ & 75 & 3 years & NA & NA & \\
\hline & & & 68 & $\mathrm{~F}$ & FNZ & 10 & 3 years & NA & NA & \\
\hline & & & 62 & $\mathrm{~F}$ & FNZ & 10 & NR & NA & NA & $\begin{array}{l}\mathrm{CH} \text { : Possible interaction with } \\
\text { verapamil }\end{array}$ \\
\hline $\begin{array}{l}\text { Benito-Leon } \\
\text { et al. }\end{array}$ & Spain 2003 & 9 & NA & NA & $\begin{array}{l}8 \mathrm{CNZ} / \\
1 \mathrm{FNZ}\end{array}$ & NA & NA & NA & NA & \\
\hline \multirow[t]{2}{*}{ Fabiani et al. } & \multirow[t]{2}{*}{ Brazil 2004} & 4 & $\begin{array}{l}61.75 \\
\text { (mean) }\end{array}$ & $\begin{array}{l}2 \mathrm{~F} \\
+ \\
2 \mathrm{M}\end{array}$ & $\begin{array}{l}2 \mathrm{FNZI} \\
2 \mathrm{CNZ}\end{array}$ & $\begin{array}{l}11.2 / 72.1 \\
\text { (mean) }\end{array}$ & $\begin{array}{l}16.5 \\
\text { months } \\
\text { (mean) }\end{array}$ & NR & NR & $\begin{array}{l}\text { CH: Only PKN. CM: Drug } \\
\text { withdrawal }\end{array}$ \\
\hline & & 1 & 87 & $\mathrm{~F}$ & $\mathrm{FNZ}+\mathrm{CNZ}$ & $10+75$ & $\begin{array}{l}16.5 \\
\text { months } \\
\text { (mean) }\end{array}$ & NR & NR & CH: PKN +orofacial DKN +DPS \\
\hline
\end{tabular}


Table 1 Clinical reports of CNZ/FNZ-associated MD (Continued)

\begin{tabular}{|c|c|c|c|c|c|c|c|c|c|c|}
\hline Reference & $\begin{array}{l}\text { Country/ } \\
\text { year }\end{array}$ & $\begin{array}{l}N \\
\text { cases }\end{array}$ & Age & Sex & $\begin{array}{l}\text { Suspected } \\
\text { drug }\end{array}$ & $\begin{array}{l}\text { Drug } \\
\text { dose } \\
\text { (mg) }\end{array}$ & $\begin{array}{l}\text { Time from } \\
\text { drug-start } \\
\text { to } \\
\text { symptoms }\end{array}$ & $\begin{array}{l}\text { Time from } \\
\text { withdrawal } \\
\text { to recovery }\end{array}$ & $\begin{array}{l}\text { Follow- } \\
\text { up }\end{array}$ & $\begin{array}{l}\text { Important clinical history and } \\
\text { clinical management }\end{array}$ \\
\hline & & 1 & 76 & $\mathrm{~F}$ & $\mathrm{FNZ}+\mathrm{CNZ}$ & $10+75$ & $\begin{array}{l}16.5 \\
\text { months } \\
\text { (mean) }\end{array}$ & NR & NR & $\mathrm{CH}: \mathrm{PKN}$ +orofacial DKN \\
\hline & & 5 & 66 (mean) & $5 F$ & $\begin{array}{l}3 \mathrm{CNZ/} \\
1 \mathrm{FNZI} \\
1 \mathrm{CNZ}+\mathrm{FNZ}\end{array}$ & $\begin{array}{l}\text { 11.2/72.1 } \\
\text { (mean) }\end{array}$ & $\begin{array}{l}16.5 \\
\text { months } \\
\text { (mean) }\end{array}$ & NR & NR & $\mathrm{CH}: \mathrm{PKN}+\mathrm{DPS}$ \\
\hline $\begin{array}{l}\text { Trevisol- } \\
\text { Bittencourt } \\
\text { et al. }\end{array}$ & Brazil 2005 & 3 & 73.2 (mean) & $\mathrm{F}$ & CNZ/FNZ & NR & NR & NR & NR & \\
\hline Barbosa et al. & Brazil 2006 & 13 & 73.5 (mean) & NR & $\begin{array}{l}7 F N Z / \\
6 C N Z\end{array}$ & NR & NR & NR & NR & \\
\hline $\begin{array}{l}\text { Louter and } \\
\text { Tromp }\end{array}$ & $\begin{array}{l}\text { Netherlands } \\
2009\end{array}$ & 1 & Adult & NR & CNZ & NR & NR & NR & $C R$ & CM: Drug withdrawal \\
\hline Ma et al. & Korea 2009 & 6 & 71.5 (mean) & NR & FNZ & NR & $\begin{array}{l}6.3 \\
\text { months } \\
\text { (mean) }\end{array}$ & NR & NR & \\
\hline Mattos et al. & Brazil 2009 & 1 & 74 & $\mathrm{~F}$ & FNZ & 10 & 1 year & 10 months & $C R$ & $\begin{array}{l}\text { CH: Progressive supranuclear } \\
\text { palsy like syndrome. CM: Drug } \\
\text { withdrawal; levodopa, } \\
\text { tolcapone, and memantine } \\
\text { were started }\end{array}$ \\
\hline $\begin{array}{l}\text { Munhoz } \\
\text { et al. }\end{array}$ & Brazil 2010 & 47 & 60.8 (mean) & NA & $\begin{array}{l}34 \mathrm{FNZI} \\
13 \mathrm{CNZ}\end{array}$ & NA & NA & NA & NA & \\
\hline $\begin{array}{l}\text { Masmoudi } \\
\text { et al. }\end{array}$ & France 2011 & 1 & 80 & $\mathrm{~F}$ & FNZ & 10 & Months & NR & No & $\begin{array}{l}\mathrm{CH}: \text { PKN +orofacial DKN; } \\
\text { possible interaction with } \\
\text { trimetazidine; she did not } \\
\text { recover the DKN }\end{array}$ \\
\hline \multirow[t]{2}{*}{ Arias } & $\begin{array}{l}\text { Colombia } \\
2012\end{array}$ & 2 & 35 & $\mathrm{~F}$ & FNZ & 20 & NR & 10 weeks & $C R$ & $\begin{array}{l}\text { CH: PKN +DPS. CM: Drug } \\
\text { withdrawal }\end{array}$ \\
\hline & & & 28 & M & FNZ & 20 & 6 weeks & 10 weeks & $C R$ & $\begin{array}{l}\text { CH: PKN +DPS. CM: Drug } \\
\text { withdrawal }\end{array}$ \\
\hline Pioner et al. & Brazil 2012 & 1 & 56 & $\mathrm{~F}$ & CNZ & 25 & NR & NR & $C R$ & CM: Drug withdrawal \\
\hline \multirow[t]{6}{*}{ Kim et al. } & Korea 2013 & 6 & 65 & $\mathrm{~F}$ & FNZ & 10 & $\begin{array}{l}12 \\
\text { months }\end{array}$ & NR & NR & CH: PKN. CM: Drug withdrawal \\
\hline & & & 62 & M & FNZ & 10 & 1 month & NR & NR & CH: PKN. CM: Drug withdrawal \\
\hline & & & 84 & $\mathrm{~F}$ & FNZ & 10 & 3 months & NR & NR & CH: PKN. CM: Drug withdrawal \\
\hline & & & 70 & F & FNZ & 10 & $\begin{array}{l}48 \\
\text { months }\end{array}$ & NR & NR & CH: PKN. CM: Drug withdrawal \\
\hline & & & 58 & $\mathrm{~F}$ & FNZ & 10 & 1 month & NR & NR & CH: PKN +oromandibular DTN \\
\hline & & & 66 & $\mathrm{~F}$ & FNZ & 10 & 3 months & NR & NR & CH: PKN. CM: Drug withdrawal \\
\hline $\begin{array}{l}\text { Gotardelo } \\
\text { et al. }\end{array}$ & Brazil 2014 & 1 & 72 & $\mathrm{~F}$ & FNZ & 10 & Years & 2 months & $C R$ & $\begin{array}{l}\text { CM: Drug withdrawal; } \\
\text { biperiden started }\end{array}$ \\
\hline Miguel et al. & $\begin{array}{l}\text { Portugal } \\
2014\end{array}$ & 30 & 73.3 (mean) & $\begin{array}{l}22 \mathrm{~F} \\
+ \\
8 \mathrm{M}\end{array}$ & FNZ/CNZ & NR & NR & NR & $\begin{array}{l}\text { CR } \\
(43 \%)\end{array}$ & $\begin{array}{l}\mathrm{CH}: 43 \% \text { recovered only with } \\
\text { withdrawal; the others needed } \\
\text { a dopaminergic treatment for } \\
\text { improving the symptoms }\end{array}$ \\
\hline $\begin{array}{l}\text { Chary and } \\
\text { Krishnan }\end{array}$ & India 2016 & 1 & 37 & $\mathrm{~F}$ & FNZ & 15 & 1 month & 1 week & $C R$ & $\begin{array}{l}\text { CH: PKN +DPS. CM: Drug } \\
\text { withdrawal; trihexyphenidyl } \\
\text { started }\end{array}$ \\
\hline $\begin{array}{l}\text { Munhoz } \\
\text { et al. }\end{array}$ & Brazil 2016 & 58 & 74.1 (mean) & NR & $\begin{array}{l}38 F N Z / \\
20 C N Z\end{array}$ & $\begin{array}{l}9.1 / 45 \\
\text { (mean) }\end{array}$ & 6 months & NR & NR & \\
\hline Nistico et al. & Italy 2016 & 2 & $\begin{array}{l}64.19 \\
\text { (mean) }\end{array}$ & $2 \mathrm{~F}$ & FNZ & NR & NR & NR & NR & \\
\hline
\end{tabular}


Table 1 Clinical reports of CNZ/FNZ-associated MD (Continued)

\begin{tabular}{|c|c|c|c|c|c|c|c|c|c|c|}
\hline Reference & $\begin{array}{l}\text { Country/ } \\
\text { year }\end{array}$ & $\begin{array}{l}N \\
\text { cases }\end{array}$ & Age & Sex & $\begin{array}{l}\text { Suspected } \\
\text { drug }\end{array}$ & $\begin{array}{l}\text { Drug } \\
\text { dose } \\
\text { (mg) }\end{array}$ & $\begin{array}{l}\text { Time from } \\
\text { drug-start } \\
\text { to } \\
\text { symptoms }\end{array}$ & $\begin{array}{l}\text { Time from } \\
\text { withdrawal } \\
\text { to recovery }\end{array}$ & $\begin{array}{l}\text { Follow- } \\
\text { up }\end{array}$ & $\begin{array}{l}\text { Important clinical history and } \\
\text { clinical management }\end{array}$ \\
\hline Sung et al. & Korea 2016 & 1 & $\begin{array}{l}70.85 \\
\text { (mean) }\end{array}$ & $1 \mathrm{~F}$ & CNZ & NR & NR & NR & NR & \\
\hline \multicolumn{11}{|l|}{ Dyskinesia } \\
\hline \multirow[t]{2}{*}{ Micheli et al. } & $\begin{array}{l}\text { Argentina } \\
1987\end{array}$ & 2 & 64 & $F$ & FNZ & 10 & 3 months & 2 months & $C R$ & $\begin{array}{l}\text { CH: Orofacial DKN (probably } \\
\text { rabbit syndrome). CM: Drug } \\
\text { withdrawal }\end{array}$ \\
\hline & & & 62 & $F$ & FNZ & 10 & 2 years & NA & No & $\begin{array}{l}\text { CH: Orofacial DKN +DPS. CM: } \\
\text { Drug withdrawal, but without } \\
\text { symptoms resolution }\end{array}$ \\
\hline $\begin{array}{l}\text { Gabellini } \\
\text { et al. }\end{array}$ & Italy 1989 & 1 & 62 & $F$ & FNZ & 10 & 1 year & 3 weeks & $C R$ & $\begin{array}{l}\text { CH: Transient tongue tremor. } \\
\text { CM: Drug withdrawal }\end{array}$ \\
\hline \multirow[t]{2}{*}{$\begin{array}{l}\text { Mangone } \\
\text { et al. }\end{array}$} & $\begin{array}{l}\text { Argentina } \\
1989\end{array}$ & 6 & 68.5 (mean) & $\begin{array}{l}5 \mathrm{~F} \\
+1 \\
\mathrm{M}\end{array}$ & FNZ/CNR & NR & NR & 2 months & NR & $\begin{array}{l}\text { CH: Orofacial DKN. CM: Drug } \\
\text { withdrawal }\end{array}$ \\
\hline & & 1 & 68.5 (mean) & $1 \mathrm{~F}$ & FNZ/CNR & NR & NR & 2 months & $C R$ & $\begin{array}{l}\text { CH: Rabbit syndrome + AKT. } \\
\text { CM: Drug withdrawal }\end{array}$ \\
\hline \multirow[t]{9}{*}{ Micheli et al. } & $\begin{array}{l}\text { Argentina } \\
1989\end{array}$ & 9 & 74 & $F$ & FNZ & 10 & $\begin{array}{l}36 \\
\text { months }\end{array}$ & NA & No & $\begin{array}{l}\text { CH: Orofacial DKN +AKT. CM: } \\
\text { Drug withdrawal }\end{array}$ \\
\hline & & & 59 & M & CNZ & 225 & $\begin{array}{l}36 \\
\text { months }\end{array}$ & 2 weeks & $C R$ & $\begin{array}{l}\text { CH: Orofacial DKN +PKN +DPS. } \\
\text { CM: Drug withdrawal }\end{array}$ \\
\hline & & & 62 & $F$ & CNZ & 150 & $\begin{array}{l}24 \\
\text { months }\end{array}$ & 1 month & $C R$ & $\begin{array}{l}\text { CH: Orofacial DKN. CM: Drug } \\
\text { withdrawal }\end{array}$ \\
\hline & & & 64 & $F$ & FNZ & 10 & 4 months & 1 month & $C R$ & $\begin{array}{l}\text { CH: Orofacial DKN +PKN. CM: } \\
\text { Drug withdrawal }\end{array}$ \\
\hline & & & 61 & $F$ & FNZ & 11.5 & 3 months & 5 months & $C R$ & $\begin{array}{l}\text { CH: Orofacial DKN +AKT +PKN } \\
\text { +DPS. CM: Drug withdrawal }\end{array}$ \\
\hline & & & 70 & $F$ & $\mathrm{FNZ}+\mathrm{CNZ}$ & $\begin{array}{l}25 / 10 \\
m g\end{array}$ & $\begin{array}{l}24 \\
\text { months }\end{array}$ & NA & No & $\begin{array}{l}\text { CH: Orofacial DKN +AKT. CM: } \\
\text { Drug withdrawal }\end{array}$ \\
\hline & & & 68 & $F$ & FNZ & 10 & $\begin{array}{l}48 \\
\text { months }\end{array}$ & 5 months & $C R$ & $\begin{array}{l}\text { CH: Orofacial DKN +PKN +DPS. } \\
\text { CM: Drug withdrawal }\end{array}$ \\
\hline & & & 64 & $F$ & FNZ & 10 & $\begin{array}{l}24 \\
\text { months }\end{array}$ & NA & No & $\begin{array}{l}\text { CH: Orofacial DKN +AKT +DPS. } \\
\text { CM: Drug withdrawal }\end{array}$ \\
\hline & & & 84 & M & CNZ & 150 & 4 months & NA & No & $\begin{array}{l}\mathrm{CH} \text { : Orofacial DKN +PKN. CM: } \\
\text { Drug withdrawal }\end{array}$ \\
\hline $\begin{array}{l}\text { Jimenez- } \\
\text { Jimenez et al. }\end{array}$ & Spain 1996 & 2 & 70 (mean) & $2 \mathrm{~F}$ & FNZ/CNR & NR & $\begin{array}{l}60.9 \\
\text { months } \\
\text { (mean) }\end{array}$ & $\begin{array}{l}4.5 \text { months } \\
\text { (mean) }\end{array}$ & $C R$ & \\
\hline $\begin{array}{l}\text { Orti-Pareja } \\
\text { et al. }\end{array}$ & Spain 1999 & 1 & 75.6 (mean) & F & FNZ & NA & $\begin{array}{l}45.8 \\
\text { months }\end{array}$ & NA & NA & \\
\hline Fabiani et al. & Brazil 2004 & 1 & 72 & M & FNZ & 10 & $\begin{array}{l}16.5 \\
\text { months } \\
\text { (mean) }\end{array}$ & NR & NR & \\
\hline \multicolumn{11}{|l|}{ Akathisia } \\
\hline Micheli et al. & $\begin{array}{l}\text { Argentina } \\
1987\end{array}$ & 1 & 54 & M & CNZ & 75 & $4 \mathrm{~h}$ & 1 day & $C R$ & CM: Drug withdrawal \\
\hline \multirow[t]{4}{*}{ Micheli et al. } & $\begin{array}{l}\text { Argentina } \\
1989\end{array}$ & 4 & 70 & $\mathrm{~F}$ & FNZ & 30 & $\begin{array}{l}48 \\
\text { months }\end{array}$ & 2 months & $C R$ & $\begin{array}{l}\mathrm{CH}: \mathrm{AKT}+\text { bruxism + PKN. CM: } \\
\text { Drug withdrawal }\end{array}$ \\
\hline & & & 49 & $\mathrm{~F}$ & FNZ & 20 & 8 months & 2 months & $C R$ & $\begin{array}{l}\mathrm{CH}: \text { AKT +PKN +DPS. CM: Drug } \\
\text { withdrawal }\end{array}$ \\
\hline & & & 66 & $\mathrm{~F}$ & FNZ & 10 & $\begin{array}{l}18 \\
\text { months }\end{array}$ & 2 months & $C R$ & $\begin{array}{l}\mathrm{CH}: \text { AKT +PKN +DPS. CM: Drug } \\
\text { withdrawal }\end{array}$ \\
\hline & & & 74 & M & FNZ & 20 & 18 & 8 months & $C R$ & CH: AKT +PKN +DPS. CM: Drug \\
\hline
\end{tabular}


Table 1 Clinical reports of CNZ/FNZ-associated MD (Continued)

\begin{tabular}{|c|c|c|c|c|c|c|c|c|c|c|}
\hline Reference & $\begin{array}{l}\text { Country/ } \\
\text { year }\end{array}$ & $\begin{array}{l}N \\
\text { cases }\end{array}$ & Age & Sex & $\begin{array}{l}\text { Suspected } \\
\text { drug }\end{array}$ & $\begin{array}{l}\text { Drug } \\
\text { dose } \\
\text { (mg) }\end{array}$ & $\begin{array}{l}\text { Time from } \\
\text { drug-start } \\
\text { to } \\
\text { symptoms }\end{array}$ & $\begin{array}{l}\text { Time from } \\
\text { withdrawal } \\
\text { to recovery }\end{array}$ & $\begin{array}{l}\text { Follow- } \\
\text { up }\end{array}$ & $\begin{array}{l}\text { Important clinical history and } \\
\text { clinical management }\end{array}$ \\
\hline & & & & & & & months & & & withdrawal \\
\hline $\begin{array}{l}\text { Garcia and } \\
\text { Uriarte }\end{array}$ & Spain 1991 & 1 & Adult & NA & FNZ & NA & NA & NA & NA & \\
\hline $\begin{array}{l}\text { Anand and } \\
\text { Thiagarajan }\end{array}$ & India 1993 & 1 & Adult & NA & FNZ & NA & NA & NA & NA & $\mathrm{CH}: \mathrm{AKT}+\mathrm{DPS}$ \\
\hline $\begin{array}{l}\text { Jimenez- } \\
\text { Jimenez et al. }\end{array}$ & Spain 1996 & 2 & 70 (mean) & $2 \mathrm{~F}$ & FNZ/CNR & NR & $\begin{array}{l}60.9 \\
\text { months } \\
\text { (mean) }\end{array}$ & $\begin{array}{l}4.5 \text { months } \\
\text { (mean) }\end{array}$ & $C R$ & \\
\hline \multicolumn{11}{|l|}{ Dystonia } \\
\hline Micheli et al. & $\begin{array}{l}\text { Argentina } \\
1987\end{array}$ & 1 & 37 & M & $\mathrm{FNZ}+\mathrm{CNZ}$ & $10+150$ & 3 days & NA & NA & $\begin{array}{l}\text { CH: Cervical DTN. CM: } \\
\text { FNZ+CNZ was maintained }\end{array}$ \\
\hline $\begin{array}{l}\text { Mangone } \\
\text { et al. }\end{array}$ & $\begin{array}{l}\text { Argentina } \\
1989\end{array}$ & 6 & 68.5 (mean) & $\begin{array}{l}1 \mathrm{~F} \\
+ \\
5 \mathrm{M}\end{array}$ & FNZ/CNR & NR & NR & NR & $C R$ & $\begin{array}{l}\text { CH: Acute DTN that resolved } \\
\text { after drug withdrawal }\end{array}$ \\
\hline Micheli et al. & $\begin{array}{l}\text { Argentina } \\
1989\end{array}$ & 1 & 67 & $\mathrm{~F}$ & $\mathrm{FNZ}+\mathrm{CNZ}$ & $20+150$ & $\begin{array}{l}18 \\
\text { months }\end{array}$ & NA & No & $\begin{array}{l}\mathrm{CH} \text { : Blepharospasm + } \\
\text { oromandibular DTN. CM: Drug } \\
\text { withdrawal }\end{array}$ \\
\hline Biary et al. & Arabia 1995 & 1 & 31 & $\mathrm{~F}$ & FNZ & 10 & 3 months & NR & NR & CH: Cervical DTN \\
\hline $\begin{array}{l}\text { Jimenez- } \\
\text { Jimenez et al. }\end{array}$ & Spain 1996 & 2 & 70 (mean) & $2 \mathrm{~F}$ & FNZ/CNR & NR & $\begin{array}{l}60.9 \\
\text { months } \\
\text { (mean) }\end{array}$ & $\begin{array}{l}4.5 \text { months } \\
\text { (mean) }\end{array}$ & $C R$ & \\
\hline $\begin{array}{l}\text { Koukoulis } \\
\text { et al. }\end{array}$ & Spain 1997 & 1 & 30 & F & FNZ & 10 & 2 months & 1 month & $C R$ & $\begin{array}{l}\text { CH: Blepharospasm. CM: Drug } \\
\text { withdrawal }\end{array}$ \\
\hline Fabiani et al. & Brazil 2004 & 1 & 61 & $\mathrm{~F}$ & $\mathrm{FNZ}+\mathrm{CNZ}$ & $\begin{array}{l}11.2 / 72.1 \\
\text { (mean) }\end{array}$ & $\begin{array}{l}16.5 \\
\text { months } \\
\text { (mean) }\end{array}$ & NA & NA & \\
\hline $\begin{array}{l}\text { Alonso- } \\
\text { Navarro and } \\
\text { Jimenez- } \\
\text { Jimenez }\end{array}$ & Spain 2006 & 1 & 53 & $\mathrm{~F}$ & CNZ & 40 & 6 years & 1 year & $C R$ & $\begin{array}{l}\text { CH: Blepharospasm; she also } \\
\text { had a history of DTN with } \\
\text { thiethylperazine and sulpiride. } \\
\text { CM: Drug withdrawal }\end{array}$ \\
\hline $\begin{array}{l}\text { Mathews } \\
\text { et al. }\end{array}$ & India 2017 & 1 & 17 & $\mathrm{~F}$ & CNZ & 25 & $\begin{array}{l}\text { Single- } \\
\text { dose }\end{array}$ & 1.5 days & $C R$ & $\begin{array}{l}\text { CH: Oromandibular and } \\
\text { cervical DTN; possible } \\
\text { interaction between CNZ and } \\
\text { prochlorperazine. CM: Drug } \\
\text { withdrawal; diphenhydramine } \\
\text { started }\end{array}$ \\
\hline Gallop et al. & UK 2019 & 1 & 10.5 (mean) & $\mathrm{F}$ & FNZ & $5 \mathrm{mg}$ & NA & NA & NA & $\begin{array}{l}\mathrm{CH} \text { : Worsening of DTN; Sturge- } \\
\text { Weber syndrome }\end{array}$ \\
\hline \multicolumn{11}{|l|}{ Myoclonus } \\
\hline Turner et al. & Israel 2006 & 1 & 2.5 & $\mathrm{~F}$ & CNZ & Overdose & NA & NA & NA & $\begin{array}{l}\mathrm{CH} \text { : Possible MCL (twitching in } \\
\text { both hands) }\end{array}$ \\
\hline \multirow{4}{*}{$\begin{array}{l}\text { Lopez- } \\
\text { Castellanos } \\
\text { et al. }\end{array}$} & \multirow[t]{4}{*}{$\begin{array}{l}\text { El Salvador } \\
2017\end{array}$} & 4 & 58 & M & $\mathrm{FNZ}+\mathrm{CNZ}$ & NR & 1 week & 3 days & $C R$ & $\begin{array}{l}\text { CH: Multifocal MCL. CM: Drug } \\
\text { withdrawal }\end{array}$ \\
\hline & & & 66 & $\mathrm{~F}$ & FNZ & $N R$ & 20 years & 1 month & $C R$ & $\begin{array}{l}\text { CH: Multifocal MCL. CM: Drug } \\
\text { withdrawal }\end{array}$ \\
\hline & & & 70 & $\mathrm{~F}$ & CNZ & NR & 8 years & NA & No & $\begin{array}{l}\text { CH: Multifocal MCL. CM: Drug } \\
\text { withdrawal }\end{array}$ \\
\hline & & & 69 & M & CNZ & NR & 3 years & 5 years & $C R$ & $\begin{array}{l}\text { CH: Multifocal MCL. CM: Drug } \\
\text { withdrawal }\end{array}$ \\
\hline
\end{tabular}

\section{Cases not clearly defined}


Table 1 Clinical reports of CNZ/FNZ-associated MD (Continued)

\begin{tabular}{|c|c|c|c|c|c|c|c|c|c|c|}
\hline Reference & $\begin{array}{l}\text { Country/ } \\
\text { year }\end{array}$ & $\begin{array}{l}N \\
\text { cases }\end{array}$ & Age & Sex & $\begin{array}{l}\text { Suspected } \\
\text { drug }\end{array}$ & $\begin{array}{l}\text { Drug } \\
\text { dose } \\
\text { (mg) }\end{array}$ & $\begin{array}{l}\text { Time from } \\
\text { drug-start } \\
\text { to } \\
\text { symptoms }\end{array}$ & $\begin{array}{l}\text { Time from } \\
\text { withdrawal } \\
\text { to recovery }\end{array}$ & $\begin{array}{l}\text { Follow- } \\
\text { up }\end{array}$ & $\begin{array}{l}\text { Important clinical history and } \\
\text { clinical management }\end{array}$ \\
\hline
\end{tabular}

\begin{tabular}{|c|c|c|c|}
\hline $\begin{array}{l}\text { Giannaula } \\
\text { et al. }\end{array}$ & $\begin{array}{l}\text { Argentina } \\
1986\end{array}$ & 27 & EPS \\
\hline Amery & $\begin{array}{l}\text { Belgium } \\
1987\end{array}$ & $>1$ & EPS \\
\hline Baldrati et al. & Italy 1987 & 1 & Tremor \\
\hline $\begin{array}{l}\text { Herskovits } \\
\text { and } \\
\text { Mangone }\end{array}$ & $\begin{array}{l}\text { Argentina } \\
1987\end{array}$ & $>1$ & EPS \\
\hline $\begin{array}{l}\text { Assmann } \\
\text { et al. }\end{array}$ & $\begin{array}{l}\text { Netherlands } \\
1988\end{array}$ & $>1$ & EPS \\
\hline di Rosa & Italy 1988 & $>1$ & EPS \\
\hline Rostin & France 1988 & $>1$ & EPS \\
\hline $\begin{array}{l}\text { Hefner and } \\
\text { Fischer }\end{array}$ & $\begin{array}{l}\text { Germany } \\
1989\end{array}$ & $>1$ & PKN \\
\hline $\begin{array}{l}\text { Jongerius } \\
\text { and van Gool }\end{array}$ & $\begin{array}{l}\text { Netherlands } \\
1989\end{array}$ & $>1$ & EPS \\
\hline $\begin{array}{l}\text { Mangone } \\
\text { et al. }\end{array}$ & $\begin{array}{l}\text { Argentina } \\
1989\end{array}$ & 8 & Tremor \\
\hline Petri & $\begin{array}{l}\text { Netherlands } \\
1989\end{array}$ & $>1$ & EPS \\
\hline $\begin{array}{l}\text { Centozone } \\
\text { et al. }\end{array}$ & Italy 1990 & 1 & Tremor \\
\hline Micheli et al. & $\begin{array}{l}\text { Argentina } \\
1990\end{array}$ & 2 & Bradykin \\
\hline Senard et al. & France 1990 & 6 & EPS \\
\hline \multirow{2}{*}{$\begin{array}{l}\text { Wilder-Smith } \\
\text { et al. }\end{array}$} & \multirow{2}{*}{$\begin{array}{l}\text { Switzerland } \\
1991\end{array}$} & 1 & Tremor \\
\hline & & 1 & AKT \\
\hline $\begin{array}{l}\text { Curran and } \\
\text { Lang }\end{array}$ & $\begin{array}{l}\text { Canada } \\
1993\end{array}$ & 3 & Tremor \\
\hline Beghi et al. & Italy1994 & $>1$ & PKN \\
\hline Brucke et al. & Austria 1995 & NA & EPS \\
\hline Martí-Masso & Spain 1996 & $>1$ & PKN \\
\hline Vecchio et al. & Italy 1996 & 3 & Tremor \\
\hline $\begin{array}{l}\text { Verspeelt } \\
\text { et al. }\end{array}$ & $\begin{array}{l}\text { Germany } \\
1996\end{array}$ & 43 & EPS \\
\hline $\begin{array}{l}\text { Orti-Pareja } \\
\text { et al. }\end{array}$ & Spain 1999 & 2 & Tremor \\
\hline $\begin{array}{l}\text { Vazquez-Alen } \\
\text { et al. }\end{array}$ & Spain 2000 & $>1$ & PKN \\
\hline $\begin{array}{l}\text { Schillevoort } \\
\text { et al. }\end{array}$ & $\begin{array}{l}\text { Netherlands } \\
2002\end{array}$ & $>1$ & PKN \\
\hline
\end{tabular}

Report of 27 individuals that developed PKN +DPS after CNZ/FNZ use

Reports about EPS following the use of FNZ

A young female presented with unilateral postural tremor after $10 \mathrm{mg}$ FNZ for 2 months. Later, 4 months, she developed DPS. No signs of PKN were observed

EPS following the use of CNZ/FNZ

EPS following the use of FNZ

EPS following the use of FNZ

Assessment of the efficacy of FNZ to the prophylactic treatment of migraine

Worsening of PD symptoms with FNZ

EPS following the use of FNZ

The symptoms started within 15 months of the beginning of the FNZ/CNZ and recovery in two months after drug withdrawal

EPS following the use of FNZ

Assessment of the efficacy of FNZ to the prophylactic treatment of migraine

Assessment of the efficacy of FNZ to the management of Tourette's syndrome

Report of $5 \mathrm{~F}+1 \mathrm{M}$ with 71.5 years (mean) who were $\mathrm{n}$ use of FNZ $11.66 \mathrm{mg}$ when the EPS occurred. The EPS appeared after 7.0 (mean) months and disappeared after 2.2 (mean) months respectively

Assessment of the efficacy of CNZ as an antiemetic for platin chemotherapy, possible interaction with metoclopramide and lorazepam

Assessment of the efficacy of FNZ in 10 patients with essential tremor. 3 individuals developed worsening of the symptoms

Pharmaco-epidemiological study about the prevalence of PKN in Italy. Exposure to FNZ, neuroleptics was observed in 8 patients

SPECT assessment in 26 individuals under FNZ/CNZ. It was observed that older age and longterm treatment are predisposing factors for EPS

Determine the prevalence of DIP in general neurology practice. During 1981-1988, the drug most often implicated was CNZ, though its relative impact decreased after

Assessment of the efficacy of FNZ in 12 patients with essential tremor. 3 individuals had worsening of tremor, in the others nothing change

Assessment of the efficacy of FNZ in vestibular vertigo and migraine

Reports of tremor following the use of CNZ (1) or FNZ (1)

To determine demographic changes in an outpatient clinic in Spain about MD. It was observed a 40\% decrease of the PKN during 1991-1998; the authors hypothesized that this occurred because of a reduction in prescriptions of CNZ/FNZ and flupentixol

Data obtained from the PHARMO-database 1986-1998. CNZ/FNZ users were more likely to receive antiparkinsonian medication than non-users. Also, the use of antiparkinsonian medication was already elevated with CNZ/FNZ low doses and increased with increasing dose and duration of use 
Table 1 Clinical reports of CNZ/FNZ-associated MD (Continued)

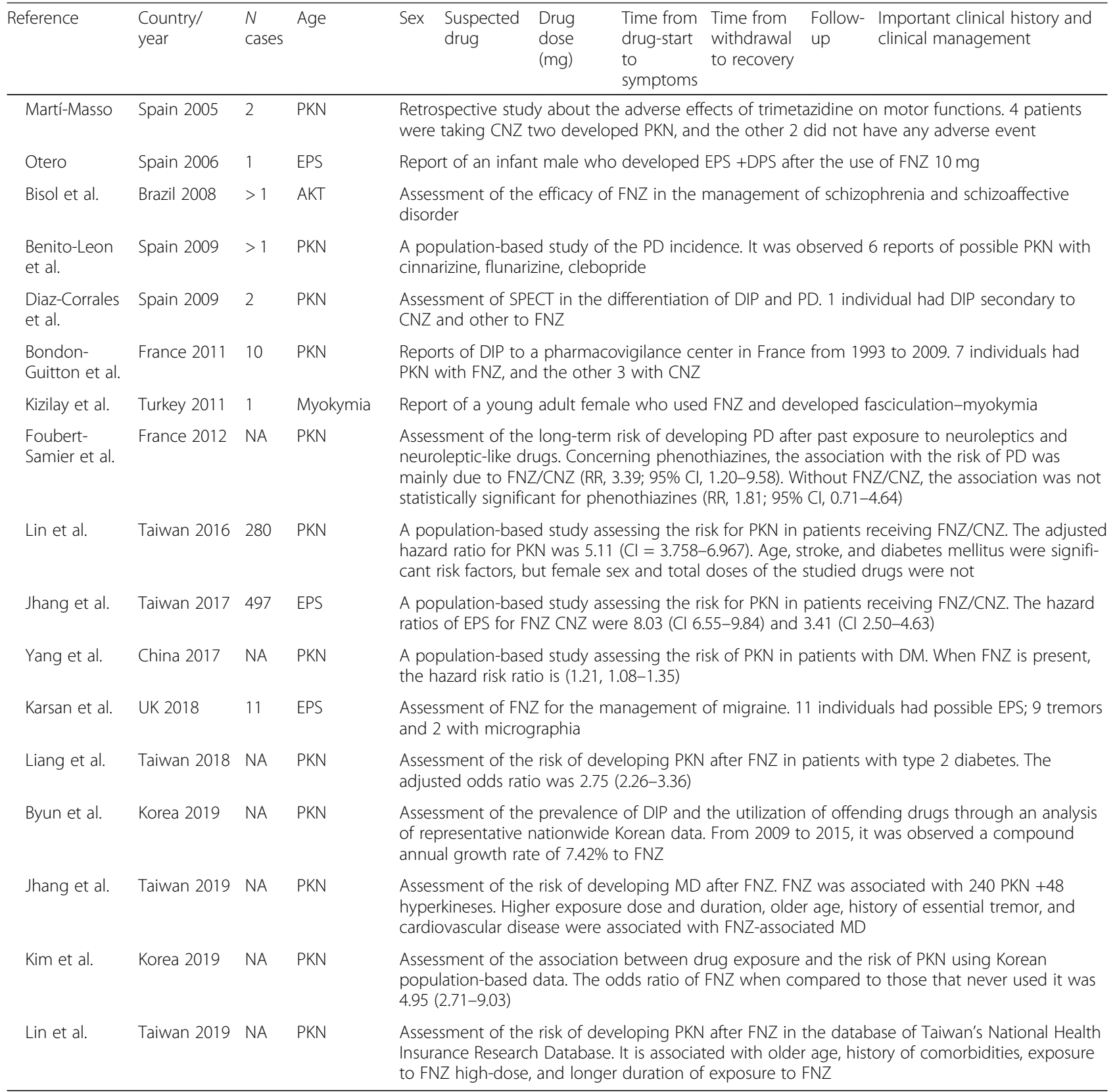

Abbreviations: $A K T$ akathisia, $B D$ bipolar disorder, $C H$ clinical history, $C M$ clinical management, $C N Z$ cinnarizine, $C R$ complete recovery, DIP drug-induced parkinsonism, DKN dyskinesia, DPS depression, DTN dystonia, EPI epilepsy, EPS extrapyramidal symptoms, $F$ female, $F N Z$ flunarizine, $M$ male, $M C L$ myoclonus, $M D$ movement disorder, NA not applicable/not available, NR not reported, PKN parkinsonism, PD Parkinson's disease, FNZ/CNZ flunarizine or cinnarizine, FNZ+CNZ flunarizine combined with cinnarizine

were 1251 parkinsonism, 23 dyskinesias, 11 akathisia, 16 dystonia, and 5 myoclonus. In the group not clearly defined, 592 were extrapyramidal symptoms, 19 tremors, 2 bradykinesia, and 1 myokymia.

The resume data about CNZ- and FNZ-associated movement disorders is provided in Table 2. Herein, we will describe the general data of all clearly defined cases.

The predominant sex was female with a percentage of $72.69 \%(466 / 641)$. The mean and median age was 74.49
(SD, 7.88) and 71.1 years (age range, $2.5-93$ years). The mean and median CNZ dose was 148.19 (SD, 42.51) and $154.4 \mathrm{mg}$ (CNZ dose range, $25-225 \mathrm{mg}$ ) and for the FNZ dose, 11.22 (5.39) and $10 \mathrm{mg}$ (FNZ dose range, 5-60).

The mean time from the CNZ/FNZ start to the MD onset was 1.83 years (SD, 1.35). About $75 \%$ of the individual had abnormal movement within 3 years of the CNZ/FNZ treatment. The mean time from the CNZ/ FNZ withdrawal until the MD recovery was 3.71 months 


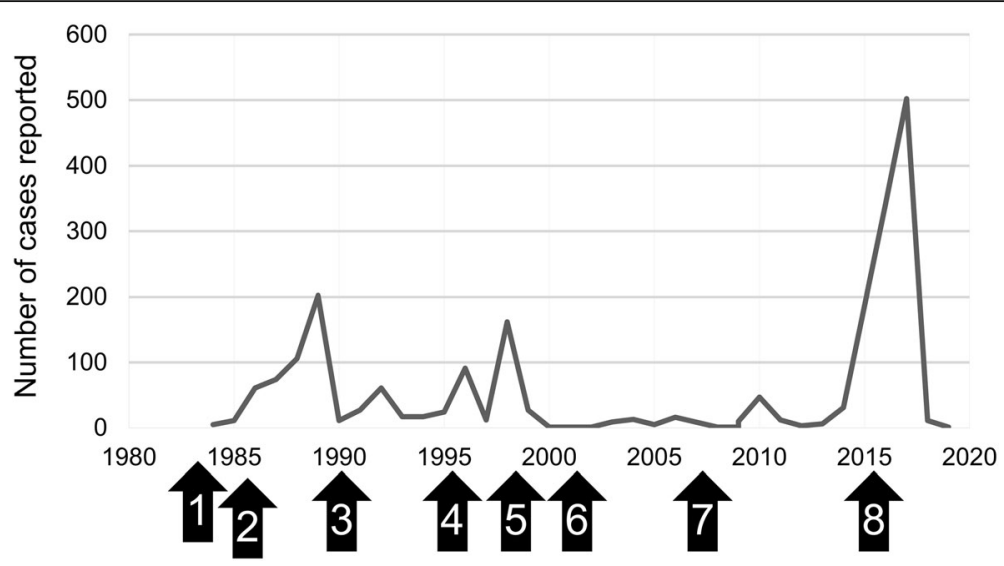

Fig. 4 Graphic showing the number of clinical reports of cinnarizine and flunarizine from 1980 to 2019. The numbers represent important markers of the history of the CNZ/FNZ-induced parkinsonism. (1) De Melo-Souza, a Brazilian neurologist, described the first to report FNZ-induced PKN. (2) Chouza and colleagues published the first report of FNZ-induced PKN. (3) Gimenez-Roldan and colleagues reported that essential tremor and older age were possible risk factors for CNZ-induced PKN. (4) Negrotti and Calzetti studied individuals with FNZ-induced PKN who had been followed for 7 years showing a bad prognosis. (5) Marti-Masso and Poza studied individuals with FNZ-induced PKN who had been followed for 10 years showing a relatively good prognosis. (6) Vazquez-Alen and colleagues showed a large decrease in drug-induced PKN; they hypothesized it was because of the reduction of CNZ/FNZ prescription. (7) Teive and colleagues called the FNZ and CNZ-induced PKN as De Melo e Souza's syndrome. (8) Population-based studies using Taiwan and Korean databases

(SD, 1.26). In the subgroup of subjects that had improvement of the symptoms, the complete recovery was achieved within 6 months of the drug withdrawal in almost all subjects (99\%). Figure 5 shows a comparison between the percentage of patients developing a MD since the beginning of the drug and the percentage of patients recovering after drug withdrawal when outliers were removed.

The most common management was drug withdrawal. Other drugs prescribed after the CNZ/FNZ withdrawal included levodopa, anticholinergics (biperiden, trihexyphenidyl, methixene, orphenadrine), benzodiazepines, propranolol, diphenhydramine, and bromocriptine. In individuals that depression was observed, amitriptyline and imipramine were one of the medications started. A complete recovery was observed in $93.77 \%$ of the patients $(437 / 466)$.

\section{Discussion}

\section{General}

Movement disorders (MD) associated with CNZ/FNZ were commonly reported in the literature. Historical facts probably have contributed to these findings such as the common sense about CNZ/FNZ be always in the list of the drugs that induced parkinsonism [131]; it was the second most common, only after antipsychotics, between the end of the 1980s and early 2000s [87]. Second, the wide number of CNZ/FNZ prescriptions all over the world because of the placebo drugs and the effect of "cerebral vasodilators" [3]. In this way, the well-known side effect and a large number of users' mixture may explain some of the reports.
Based on the data available in Table 1, we can hypothetically illustrate a case. An elderly Asian female presented with symptoms of vestibular vertigo to her general practitioner. The physician started flunarizine $10 \mathrm{mg}$. In the long-term follow-up, within about 3 years of the beginning of FNZ, she complained of slow movements, stiffness, and resting tremor. She was diagnosed with drug-induced parkinsonism, and FNZ was withdrawn. Within less than 6 months, the patient had a full recovery.

The number of reports with FNZ was more than $60 \%$ of the overall data. Two characteristics of the metabolism of FNZ, when compared to CNZ, that can explain this are the long half-life, which is more than ten times the $\mathrm{CNZ}$, and the accumulation in the central nervous system that is due to the fluorination; FNZ is much more lipophilic than CNZ [3, 6]. Moreover, we believe that another important aspect was the marketing issues with the general major availability of flunarizine all over the world [3].

The majority of the incidences of abnormal movements associated with CNZ/FNZ were not well described in the literature. Table 3 is a resume of the percentages of $\mathrm{MD}$ secondary to $\mathrm{CNZ} / \mathrm{FNZ}$; the data was extracted from clinical trials and other populationbased studies $[26,34,48,49,55,70,76,77,97,115,119$, $123,127,129]$. The incidences of CNZ/FNZ-induced abnormal movements, in general, vary throughout the literature, but the range is much smaller than other drugs with postmarketing evaluation such as valproic acid [132]; for example, extrapyramidal symptoms were found with the use of CNZ/FNZ in Verspeelt and 
Table 2 Resume of CNZ/FNZ-associated movement disorders

\begin{tabular}{|c|c|c|c|c|c|c|c|c|}
\hline Movement disorder & & PKN & DKN & AKT & DTN & $\mathrm{MCL}$ & Others & General data \\
\hline Cases (\%) & & $\begin{array}{l}1251 \\
(65.15)\end{array}$ & $23(1.19)$ & $11(0.57)$ & $16(0.83)$ & $5(0.26)$ & $\begin{array}{l}614 \\
(31.97)\end{array}$ & 1920 \\
\hline \multirow[t]{4}{*}{ Continent (\%) } & Asian & $332(26.53)$ & $0(0)$ & $1(9.09)$ & $2(12.5)$ & $1(20)$ & $\begin{array}{l}498 \\
(81.10)\end{array}$ & 834 \\
\hline & European & $575(45.96)$ & $4(17.39)$ & $4(36.36)$ & $5(31.25)$ & $0(0)$ & $\begin{array}{l}75 \\
(12.21)\end{array}$ & 663 \\
\hline & $\begin{array}{l}\text { North } \\
\text { America }\end{array}$ & $1(0.07)$ & $0(0)$ & $0(0)$ & $0(0)$ & $4(80)$ & $\begin{array}{l}3 \\
(0.48)\end{array}$ & 8 \\
\hline & $\begin{array}{l}\text { South } \\
\text { America }\end{array}$ & $343(27.41)$ & $19(82.60)$ & $6(54.54)$ & $9(56.25)$ & $0(0)$ & $\begin{array}{l}38 \\
(6.18)\end{array}$ & 415 \\
\hline \multirow[t]{3}{*}{ Sex (\%) } & Female & $429(34.29)$ & $19(82.60)$ & $5(45.45)$ & $10(62.5)$ & $3(60)$ & NA & 466 \\
\hline & Male & $161(12.86)$ & $4(17.40)$ & $2(18.18)$ & $6(37.5)$ & $2(40)$ & & 175 \\
\hline & Unknown & $661(52.83)$ & $0(0)$ & $4(36.36)$ & $0(0)$ & $0(0)$ & & 1294 \\
\hline \multirow[t]{2}{*}{ Age (years) } & Range & 25-93 & $59-84$ & $49-74$ & $10.5-70$ & $2.5-70$ & & 2.5-93 (Md, 71.1) \\
\hline & Mean & 75.63 & 67.87 & 64.71 & 53.59 & 53.1 & & 74.49 (SD, 7.88) \\
\hline \multirow[t]{4}{*}{$\begin{array}{l}\text { Number of reports with the } \\
\text { drug (mean dose in } \mathrm{mg} \text { ) }\end{array}$} & CNZ & $\begin{array}{l}347 \\
(150.12)\end{array}$ & $3(175)$ & $1(75)$ & $2(32.5)$ & $3(\mathrm{NA})$ & & $\begin{array}{l}356 \text { (Mn, 148.19; SD, 42.51; Md, 154.4; } \\
\text { Rg, 25-225) }\end{array}$ \\
\hline & FNZ & $570(11.19)$ & $10(10.16)$ & $6(20)$ & $3(8.33)$ & $1(\mathrm{NA})$ & & $\begin{array}{l}590(\mathrm{Mn}, 11.22 ; \mathrm{SD}, 5.39 ; \mathrm{Md}, 10 ; \mathrm{Rg} \\
5-60)\end{array}$ \\
\hline & $\mathrm{FNZ}+\mathrm{CNZ}$ & $\begin{array}{l}18(12.08 \\
+133.06)\end{array}$ & $\begin{array}{l}1(10+ \\
25)\end{array}$ & 0 & $\begin{array}{l}3(13.73+ \\
124.03)\end{array}$ & $1(\mathrm{NA})$ & & $\begin{array}{l}23(\mathrm{Mn}, 12.19+126.91 ; \mathrm{SD}, 2.91+ \\
39.80 ; \mathrm{Rg}, 8.75-20+25-154.4)\end{array}$ \\
\hline & $\begin{array}{l}\text { Unknown } \\
\text { CNZ/FNZ }\end{array}$ & 316 & 9 & 14 & 8 & 0 & & 951 \\
\hline \multirow[t]{2}{*}{ MD onset } & Range & $\begin{array}{l}2 \text { days }-5 \\
\text { years }\end{array}$ & $\begin{array}{l}3 \\
\text { months- } \\
5 \text { years }\end{array}$ & $\begin{array}{l}1 \text { day }-5 \\
\text { years }\end{array}$ & $\begin{array}{l}1 \text { day }-6 \\
\text { years }\end{array}$ & $\begin{array}{l}1 \text { week- } \\
20 \text { years }\end{array}$ & & 1 day-20 years (Md, 1.25) \\
\hline & $\begin{array}{l}\text { Mean } \\
\text { (years) }\end{array}$ & 1.74 & 2.21 & 2.54 & 2.46 & 7.75 & & Mn, 1.83 (SD, 1.35) \\
\hline \multirow[t]{2}{*}{ MD recovery } & Range & $\begin{array}{l}7 \text { days }-10 \\
\text { months }\end{array}$ & $\begin{array}{l}2 \text { weeks- } \\
5 \text { months }\end{array}$ & $\begin{array}{l}1 \text { day- } 8 \\
\text { months }\end{array}$ & $\begin{array}{l}1.5 \text { days- } \\
1 \text { year }\end{array}$ & $\begin{array}{l}3 \text { days }-5 \\
\text { years }\end{array}$ & & 1 day-5 years (Md, 3 months) \\
\hline & $\begin{array}{l}\text { Mean } \\
\text { (months) }\end{array}$ & 3.61 & 2.45 & 3.29 & 4.41 & 20.36 & & $3.71(S D, 1.26)$ \\
\hline \multicolumn{2}{|c|}{ Follow-up, \% CR (number of reports) } & $\begin{array}{l}94.85 \% \\
(406 / 428)\end{array}$ & $\begin{array}{l}66.66 \% \\
(10 / 15)\end{array}$ & $\begin{array}{l}100 \%(7 / \\
7)\end{array}$ & $\begin{array}{l}91.66 \% \\
(11 / 12)\end{array}$ & $\begin{array}{l}75 \%(3 / \\
4)\end{array}$ & & $93.77 \%(437 / 466)$ \\
\hline
\end{tabular}

Abbreviations: AKT akathisia, CR complete recovery, DKN dyskinesia, DTN dystonia, MCL myoclonus, MD movement disorder, Md median, Mn mean, NA not available/not applicable, $P K N$ parkinsonism, SD standard deviation, $R g$ range (minimum-maximum). In the "Others" subgroup are cases not specified about the movement disorder such as extrapyramidal symptoms, tremor, bradykinesia, and myokymia

colleagues in $4.30 \%$ of individuals with migraine individuals, but in the vestibular vertigo subgroup it was $0.91 \%$ [77].

Herein, we would like to discuss some of the MD in subtopics to give a better comprehension of the data.

\section{Parkinsonism (PKN) \\ History (Fig. 4)}

The first report of FNZ-induced PKN was by the Brazilian neurologist De Melo-Souza during the IX Brazilian Congress of Neurology in 1984 [13]. His description of five elderly females who presented PKN and depression was a crucial observation for the knowledge of druginduced MD [131]. Even though today, the number of cases has decreased and, in many countries, it has restricted prescriptions [2]; FNZ is a widely recognized drug as a cause of secondary abnormal movements and can be an example, as well as antipsychotics, for the MD. Nowadays, this association has been called De Melo e Souza's syndrome by some authors [130]. It is worthy of mentioning that Martí-Massó and colleagues in 1985 published the first report of CNZ-induced PKN [14].

\section{Incidence}

The incidence of PKN with CNZ/FNZ is scarce in the CNZ/FNZ label is that the occurrence is in 1 every 1000 users [6]; in the literature from data extraction of clinical trials, it is $0.07-6.52 \%$ (Table 3 ). It is noteworthy that the prescription of CNZ/FNZ should be avoided in PD. Martí-Massó and colleagues reported that $40 \%$ of PD 

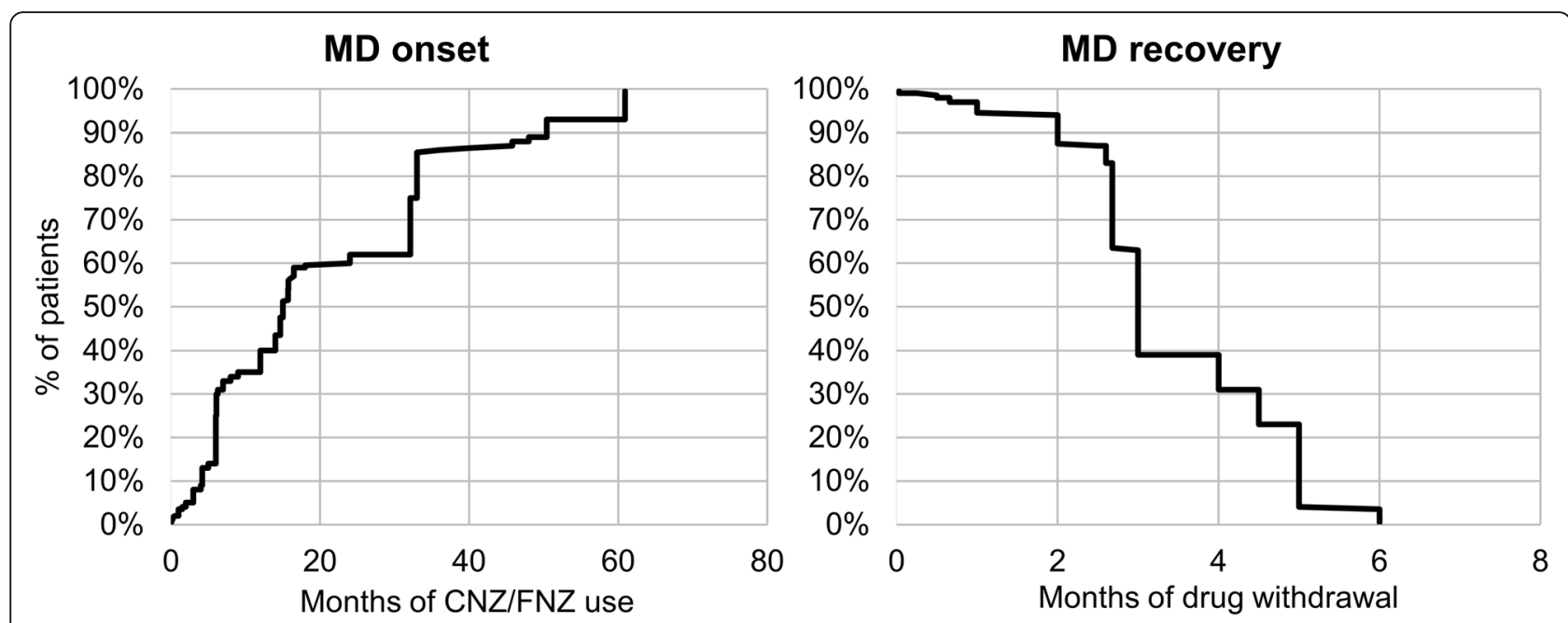

Fig. 5 Comparison between the percentage of patients developing movement disorders (MD) since the beginning of the drug and the percentage of patients recovering after drug withdrawal. The data is from 663 (MD onset) and 473 (MD recovery) individuals reported in the literature

patients with short-term CNZ/FNZ use showed a severe worsening of the bradykinesia and gait [26].

Recent Asian population-based studies showed important features of the long-term CNZ/FNZ use and its complications [115, 119, 122, 124, 127-129]. Lin et al. [115] revealed that age, stroke, and diabetes mellitus are risk factors for the development of CNZ/FNZ-induced PKN. Kim et al. [128] showed that the odds ratio of the risk of developing PKN in FNZ users when compared to non-FNZ users is 4.95 (2.719.03). In another study, Lin et al. [129] revealed that a longer duration of exposure to FNZ and high FNZ doses are significantly associated with the occurrence of PKN.

\section{Epidemiology and diagnosis}

Among the CNZ/FNZ-induced MD, PKN was the most frequently described corresponding for more than half of the cases. The majority of the individuals affected were European, and mainly from Spain probably due to a large number of prescriptions and older European populations [3]. Three epidemiological findings of this

Table 3 Incidence of some abnormal movements in the literature

\begin{tabular}{|c|c|c|c|c|c|c|c|}
\hline $\mathrm{MD}$ & Drug & Reference & Year & NR & $N$ & Incidence & Studied disease \\
\hline PKN & CNZ & Martí-Masso et al. & 1987 & 4 & 10 & $40 \%$ & Parkinson's disease \\
\hline PKN & FNZ & Martinez-Lage & 1988 & 1 & 1435 & $0.07 \%$ & Migraine \\
\hline Tremor & FNZ & Centozone et al. & 1990 & 1 & 40 & $2.50 \%$ & Migraine \\
\hline Bradykinesia & FNZ & Micheli et al. & 1990 & 2 & 7 & $28.57 \%$ & Tourette's syndrome \\
\hline Tremor & CNZ & Wilder-Smith et al. & 1991 & 1 & 14 & $7.10 \%$ & Emesis \\
\hline AKT & CNZ & Wilder-Smith et al. & 1991 & 1 & 14 & $7.10 \%$ & Emesis \\
\hline PKN & FNZ & Handforth et al. & 1995 & 1 & 16 & $6.25 \%$ & Epilepsy \\
\hline EPS & FNZ & Biary et al. & 1995 & 2 & 17 & $11.76 \%$ & Essential tremor \\
\hline Tremor & FNZ & Vecchio et al. & 1996 & 3 & 12 & $25 \%$ & Essential tremor \\
\hline EPS & FNZ & Verspeelt et al. & 1996 & 36 & 837 & $4.30 \%$ & Migraine \\
\hline EPS & FNZ & Verspeelt et al. & 1996 & 7 & 764 & $0.91 \%$ & Vestibular vertigo \\
\hline PKN & FNZ/CNZ & Lin et al. & 2016 & 280 & 9830 & $2.90 \%$ & Several \\
\hline EPS & FNZ & Jhang et al.* & 2017 & - & - & $21.03 \%$ & Several \\
\hline EPS & CNZ & Jhang et al..* & 2017 & - & - & $10.30 \%$ & Several \\
\hline Tremor & FNZ & Karsan et al. & 2018 & 9 & 200 & $4.50 \%$ & Migraine \\
\hline Micrographia & FNZ & Karsan et al. & 2018 & 2 & 200 & $1 \%$ & Migraine \\
\hline PKN & FNZ & Lin et al.* & 2019 & - & - & $8.72 \%$ & Migraine \\
\hline
\end{tabular}

Abbreviations: AKT akathisia, CNZ cinnarizine, EPS extrapyramidal symptoms, FNZ flunarizine, $N$ number of individuals in the study, NR number of reports with the movement disorder, PKN parkinsonism

*Jhang et al. incidence rate (per 10,000 person-months); Lin et al. incidence rate (per 1000 person-years) 
subgroup are comparable to the drug-induced PKN of the literature. First, the high incidence in females that is believed to be more susceptible [21], or we can presuppose that this was an occasional finding because females are related to a higher number of prescriptions. The results of Lin et al. that the female sex was not significantly associated with CNZ/FNZ treatment can support this hypothesis [115]. Second, the CNZ/FNZ-induced PKN occurred with higher CNZ/FNZ doses, which may be explained by higher doses leading to higher concentration in the central nervous system and a possible predisposition for the development of this MD [3, 6]. Third, an elderly population was more involved in this subgroup than in other abnormal movements that could be related to aging causing striatum abnormalities [9]; also, the possibility of FNZ/CNZ provoking PD cannot be ruled out.

The presentation in the majority of the cases was a symmetric akinetic-rigid syndrome, with resting and/or postural tremor; in almost half of the individuals, depression (mild, moderate, and severe) was described. Other commonly associated MD in descending order of frequency were akathisia, dyskinesia (orofacial, rabbit syndrome, choreoathetotic), and dystonia (oromandibular). Moreover, Mattos and colleagues reported a patient presenting with progressive supranuclear palsy like syndrome [102].

Sometimes, it can be hard to clearly distinguish between the CNZ/FNZ-induced MD and the idiopathic PD based only on clinical criteria. Thus, Teive et al. [131] selected some clinical tools from the studies of Negrotti and Calzetti [79] and Martí-Massó and Poza [73] to help on the diagnosis of this syndrome. Table 4 has the features by Teive et al. [131], and we propose a supporting feature that is the presence of another MD at the presentation; since an important percentage of the individuals is commonly affected by another disorder.

\section{Pathophysiological mechanism}

It is still not completely understood, but some authors believe that it is due to the decrease of dopaminergic neurotransmission [131]. In animal models, CNZ/FNZ decreased the concentration levels of dopamine probably due to tyrosine hydroxylase inhibition and dopaminergic neuron loss $[8,10]$; also, the blockage of striatal dopaminergic receptors was observed [9]. We hypothesized that the calcium-calmodulin complex inhibition by CNZ/FNZ may be involved with the decrease of dopamine [11]; the involvement of only the release of dopamine without affecting its concentration or the noradrenaline/adrenaline concentration can support this assumption [10]. Furthermore, in the literature, studies have shown a decrease [10] and/or increase [7] in serotonin concentration with CNZ/FNZ; so, the
Table 4 Clinical tools for the diagnosis of CNZ/FNZ-induced PKN by Teive et al. [131] modified by Rissardo and Caprara

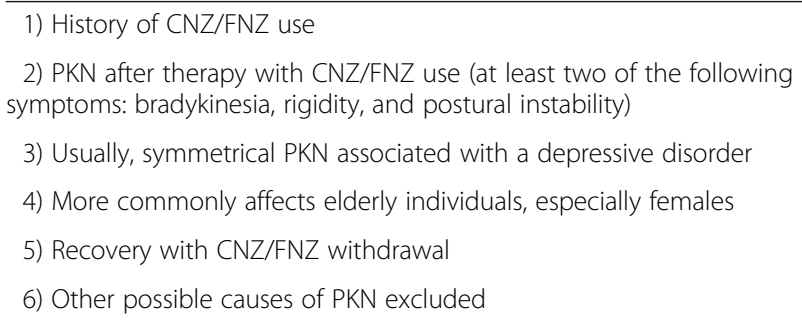

*Supporting feature: Presentation of PKN associated with other movement disorders at the same time with descending order of probability: akathisia, dyskinesia, dystonia, and possible myoclonus

CNZ cinnarizine, FNZ flunarizine, $P K N$ parkinsonism

serotoninergic hypothesis of PKN caused by a mechanism similar to that observed with serotonin reuptake inhibitors could have occurred in susceptible individuals [133].

\section{Management}

The most frequent management was the drug withdrawal and the rechallenge was not attempted in any of the cases. Some authors recommended the use of anticholinergics to accelerate the recovery and decrease the number of complications [42, 47]. A full recovery was observed in more than $90 \%$ of the cases.

\section{Dyskinesia (DKN)}

DKN was the second most commonly encountered MD secondary to CNZ/FNZ. More than $80 \%$ of the cases occurred in countries of South America. This can be explained by the great knowledge of this association leading to a possible more minute observation of the clinical findings [131]. The more frequent affected individuals were females 8 years younger than those developing PKN. Also, it was three times more common with FNZ than CNZ.

The presentation more frequently was orofacial. Rabbit syndrome was observed [15], which is an extrapyramidal adverse effect of antipsychotic medicines that perioral tremors occur at a rate of $4-5 \mathrm{~Hz}$; we included in DKN, but there is controversy in the literature and some authors believed that is a separated disorder, which goes beyond the aim of this review. Gabellini and colleagues reported isolated tongue tremors, which was rarely observed in the cases reported in the literature [39].

The most common management was drug withdrawal. However, among the CNZ/FNZ-induced MD, DKN associated with CNZ/FNZ had the worst prognosis with a full recovery obtained in only $66 \%$ of the individuals.

One of the possible explanations for the occurrence of this MD is the dopaminergic hypothesis, which happens due to an abnormal adaptation of the striatal organization leading to overactivation of the direct pathway [134]. This hypothesis is plausible in the cases 
reported and can be supported by the long onset time longer than the average. Moreover, the interaction of CNZ/FNZ with the histaminergic neurotransmission could have contributed to the development of this abnormal movement [6]. The $\mathrm{H} 1$ histamine receptors are commonly found in the tuberomammillary nucleus that has many connections with the cerebral cortex, neostriatum, hypothalamus, hippocampus, and nucleus accumbens [135]. Therefore, we believe that the long-term use of the medication can explain some of the cases, in a similar way to other antihistaminic drugs.

\section{Dystonia (DTN)}

DTN was observed in 16 individuals, and more than half was reported by South American authors. Some features of this subgroup that are commonly found in the druginduced DTN literature include the prevalence of female sex predominance, affected younger population (compared to general data), low CNZ/FNZ doses associated with DTN, and short MD onset. The presentation in descending order of frequency was blepharospasm, oromandibular, and cervical; even worsening of a previous DTN was observed in a child with Sturge-Weber syndrome, when FNZ $5 \mathrm{mg}$ was prescribed [126].

The dopaminergic hypothesis can explain the occurrence of CNZ/FNZ-induced DTN. The finding that antipsychotics that also interact with D2 dopamine receptors and are associated with DTN can support this assumption [134]. It has been suggested that the blockage of these receptors in the caudate, putamen, and globus pallidus is partly responsible for causing this abnormal movement [136]. Therefore, it is probably the disbalance of ratio dopamine-acetylcholine especially in the striatum that can produce these symptoms [9].

The most common management was drug withdrawal; in one case, diphenhydramine was started [121]. The complete recovery was noted in $91.66 \%$ of the patients.

\footnotetext{
Akathisia (AKT)

The majority of the reports associated with AKT occurred with higher FNZ doses that were almost twice the mean data. This MD most commonly occurred in the female sex from South America origin. Interesting, AKT was the only MD associated with CNZ/FNZ that after the management $100 \%$ of the users had recovered, which we believed that probably occurred because the population affected was 10 years younger than the other MD. The most frequent management was drug withdrawal. Micheli and colleagues reported the first case of CNZ/FNZ-induced AKT; a middle-age male showed AKT symptoms after the first dose of CNZ $75 \mathrm{mg}$; the drug was withdrawn and the individual recovered in one day [28].
}

Since the CNZ/FNZ is involved with the dopaminergic system; this hypothesis can feasibly explain all extrapyramidal symptoms. Therefore, as well as PKN, DKN, and DTN, the D2 dopamine block is probably related to the occurrence of AKT [134].

\section{Myoclonus (MCL)}

This MD was rarely reported in the literature in association with CNZ/FNZ. Turner and colleagues reported a case of CNZ overdose in a child, she developed twitching in both hands [95]. However, they did not provide a clear description of the neurological examination neither of the electrodiagnostic studies. In another case series, Lopez-Castellanos and Lopez-Contreras reported four subjects in 2017 at the 1st Pan American Parkinson's Disease and Movement Disorders Congress [120]. The individuals were analyzed by a movement disorder specialist but no description of electromyography or electroencephalogram was done. The presentation was multifocal with or without a tremor. The management was drug withdrawal. Only one individual did not recover after 5 years of follow-up. Based on these two reports, we cannot conclude the source of MCL.

In the literature about drug-induced MCL, the most common hypothesis is associated with the serotoninergic neurotransmission. This abnormal movement was already reported with the increase and the decrease of serotonin concentration [137]. In this context, CNZ/ FNZ was first believed to decrease the serotonin concentration, in the synaptic, by the induction of monoaminergic neuron damage probably because the higher number of reports with depressive symptoms in the clinical practice [10], but some studies have shown a contradictory increase of the serotonin that happened due to serotonin reuptake blockage and facilitation of its release [7]. We believed that these different results could have occurred due to different brain sites being studied. Therefore, the increase of this neurotransmitter may be related to MCL in susceptible individuals.

\section{Conclusion}

In sum, CNZ/FNZ-associated movement disorders were extensively reported in the literature probably due to important historical features. The most frequent and welldescribed MD was PKN. MCL was the poorest described MD with missing data about the neurological examination and electrodiagnostic studies. In descending order of frequency, the following MD related to CNZ/FNZ were encountered: $\mathrm{PKN}>\mathrm{DKN}>\mathrm{DTN}>\mathrm{AKT}>\mathrm{MCL}$. Most of the CNZ/FNZ-induced MD can be explained by the dopaminergic hypothesis, except MCL that is probably associated with serotonin. We believe that the knowledge of the abnormal movements associated with CNZ/FNZ could significantly raise the awareness of the 
potential motor side effects secondary to other commonly prescribed drugs. In this way, the continuum development of new drugs with fewer or less severe side effects is essential for the improvement of the quality of life, reduction of negative outcomes, and increase of patients' adherence.

\section{Supplementary information}

Supplementary information accompanies this paper at https://doi.org/10. 1186/s41983-020-00197-w.

Additional file 1: Other 1 - FreeText and MeSH search terms in the US National Library of Medicine

\section{Acknowledgements}

Not applicable.

\section{Authors' contributions}

JPR and ALFC contributed equally to the research idea, data acquisition, data analysis, interpretation, and manuscript review. Both authors read and approved the final manuscript.

\section{Funding}

This research received no specific grant from any funding agency in the public, commercial, or not-for-profit sectors.

\section{Availability of data and materials}

The datasets generated and/or analyzed during the current study are publicly available throughout the text and in the tables.

\section{Ethics approval and consent to participate}

Not applicable.

\section{Consent for publication}

Not applicable.

\section{Competing interests}

The authors declare that they have no competing interests.

Received: 16 January 2020 Accepted: 17 June 2020

Published online: 26 June 2020

\section{References}

1. Church MK, Gradidge CF. Inhibition of histamine release from human lung in vitro by antihistamines and related drugs. Br J Pharmacol. 1980;69(4):663-7.

2. Kirtane MV, Bhandari A, Narang P, Santani R. Cinnarizine: a contemporary review. Indian J Otolaryngol Head Neck Surg. 2019;71(2):1060-8.

3. Laporte JR, Capella D. Useless drugs are not placebos: lessons from flunarizine and cinnarizine. Lancet. 1986:2(8511):853-4.

4. Capellà D, Laporte JR, Castel JM, Tristán C, Cos A, Morales-Olivas FJ. Parkinsonism, tremor, and depression induced by cinnarizine and flunarizine. BMJ. 1988;297(6650):722-3

5. Herskovits E, Mangone C. Efectos adversos de tipo extrapiramidal y depresivo inducidos por cinarizina y flunarizina. Rev neurol Argent. 1987; 13(2):131-2.

6. Holmes B, Brogden R, Heel R, Speight T, Avery G. Flunarizine Drugs. 1984; 27(1):6-44.

7. Pukhal'skaya TG, Kolosova OA, Men'shikov MY, Vein AM. Effects of calcium antagonists on serotonin-dependent aggregation and serotonin transport in platelets of patients with migraine. Bull Exp Biol Med. 2000;130(7):633-5.

8. Takada M, Kono T, Kitai ST. Flunarizine induces a transient loss of tyrosine hydroxylase immunoreactivity in nigrostriatal neurons. Brain Res. 1992;590(12):311-5.

9. Belforte JE, Magariños-Azcone C, Armando I, Buño W, Pazo JH. Pharmacological involvement of the calcium channel blocker flunarizine in dopamine transmission at the striatum. Parkinsonism Relat Disord. 2001;8(1): $33-40$.
10. Mena MA, Garcia de Yébenes MJ, Tabernero C, Casarejos MJ, Pardo B, Garcia de Yébenes J. Effects of calcium antagonists on the dopamine system. Clin Neuropharmacol. 1995;18(5):410-26.

11. Kubo K, Matsuda Y, Kase H, Yamada K. Inhibition of calmodulin-dependent cyclic nucleotide phosphodiesterase by flunarizine, a calcium-entry blocker. Biochem Biophys Res Commun. 1984;124(2):315-21.

12. Meyboom RH, Ferrari MD, Dieleman BP. Parkinsonism, tardive dyskinesia, akathisia, and depression induced by flunarizine. Lancet. 1986;2(8501):292.

13. De Melo-Souza S. Flunarizina, parkinsonismo e depressão. Goiania, Brazil: XI Brazilian neurology meeting; 1984.

14. Martí Massó JF, Carrera N, de la Puente E. Parkinsonism possibly caused by cinnarizine. Med Clin (Barc). 1985;85(15):614-6.

15. Chouza C, Scaramelli A, Caamaño J, De Medina O, Aljanati R, Romero S. Parkinsonism, tardive dyskinesia, akathisia, and depression induced by flunarizine. Lancet. 1986;1(8493):1303-4.

16. Jankovic J, Tolosa E. Parkinson's disease and movement disorders: Lippincott Williams \& Wilkins; 2007.

17. Association AP. Diagnostic and statistical manual of mental disorders (DSM$\left.5^{\circledR}\right)$ : American Psychiatric Pub; 2013.

18. Naranjo CA, Busto U, Sellers EM, Sandor P, Ruiz I, Roberts EA, et al. A method for estimating the probability of adverse drug reactions. Clin Pharmacol Ther. 1981;30(2):239-45.

19. de Vries E, Schoonvelde M, Schumacher G. No longer lost in translation: evidence that google translate works for comparative bag-of-words text applications. Polit Anal. 2018;26(4):417-30.

20. D'Alessandro R, G B, G M. Side-effects of flunarizine. Lancet. 1986;2(8504): 463-464.

21. R Giannaula, Micheli F, Fernandez Pardal MM. Reacciones extrapiramidales inducidas por flunarizina y cinnarizina. Abstracts of the XXVI Argentine meeting of Neurology, San Miguel de Tucuman, Argentina, October 22-25. 1986.

22. Martí Massó JF. Cinarizine and Parkinson's disease. Double-blind placebocontrolled study. Neurologia. 1986;1(2):55-7.

23. Amery WK. Extrapyramidal symptoms and flunarizine. Funct Neurol. 1987; 2(3):363-4.

24. Baldrati A, Albani F, de Carolis P, Sacquegna T. Essential tremor and flunarizine: description of a case. Cephalalgia. 1987;7(4):285.

25. Di Rosa AE, Morgante L, Meduri M, Musolino R, Leggiadro N, Coraci M, et al. Parkinson-like side effects during prolonged treatment with flunarizine. Funct Neurol. 1987;2(1):47-50.

26. Martí Massó JF, Obeso JA, Carrera N, Martínez-Lage JM. Aggravation of Parkinson's disease by cinnarizine. J Neurol Neurosurg Psychiatry. 1987;50(6):804-5.

27. De Michele G, Filla A, Coppola N, Mansi D, Brescia M, Buscaino GA Extrapyramidal side-effects of flunarizine. Acta Neurol (Napoli). 1987;9(3): 230-3.

28. Micheli F, Pardal MF, Gatto M, Torres M, Paradiso G, Parera IC, et al. Flunarizine- and cinnarizine-induced extrapyramidal reactions. Neurology. 1987:37(5):881-4.

29. Assmann VC, Perquin W, Touw DJ. Extrapyramidal movement disorders following the use of flunarizine. Ned Tijdschr Geneeskd. 1988;132(42):1940-3.

30. Bakchine S, Lacomblez L, Soubrié C. Extrapyramidal syndrome during treatment with flunarizine. Rev Neurol (Paris). 1988;144(12):833-4.

31. Benvenuti F, Baroni A, Bandinelli S, Ferrucci L, Corradetti R, Pantaleo T. Flunarizine-induced parkinsonism in the elderly. J Clin Pharmacol. 1988; 28(7):600-8

32. Lugaresi A, Montagna P, Gallassi R, Lugaresi E. Extrapyramidal syndrome and depression induced by flunarizine. Eur Neurol. 1988;28(4):208-11.

33. De Marco P, Rossi G. Flunarizine and parkinson-like syndrome in a young man. Boll Lega Hal Epilessia, 62. 1988;63:227.

34. Martínez-Lage JM. Flunarizine (Sibelium) in the prophylaxis of migraine. An open, long-term, multicenter trial. Cephalalgia. 1988;8(8_suppl):15-20.

35. Moretti A, Lucantoni C. Flunarizine-induced parkinsonism: clinical report. Ital J Neurol Sci. 1988;9(3):295-7.

36. Di Rosa A. Extrapyramidal symptoms and flunarizine. Funct Neurol. 1988; 3(2):237-8.

37. Rostin M, Montastruc JL, Guiraud-Chaumeil B, Rascol A. Outcome of an extrapyramidal syndrome during prophylactic treatment of migraine with flunarizine. Therapie. 1988;43(3):242

38. Fontanari JL. A side effect of flunarizine: severe parkinsonism. Ara Neuropsiquiatr. 1989;47(3):352-4.

39. Gabellini AS, Martinelli P, Coccagna G. Drug-induced tremor of the tongue. Ital J Neurol Sci. 1989;10(1):89-91. 
40. Hefner R, Fischer PA. Increase in Parkinson symptoms caused by calcium antagonists. Nervenarzt. 1989;60(3):187-8.

41. Jongerius AM, van Gool AR. Extrapyramidal movement disorders following administration of flunarizine. Ned Tijdschr Geneeskd. 1989;133(14):746-7.

42. Kuzuhara S, Kohara N, Ohkawa Y, Fuse S, Yamanouchi H. Parkinsonism, depression and akathisia induced by flunarizine, a calcium entry blockade-report of 31 cases. Rinsho Shinkeigaku. 1989;29(6):681-6.

43. Mangone CA, Herskovits E. Extrapyramidal and depressive side reactions with flunarizine and cinarizine. J Neurol Neurosurg Psychiatry. 1989;52(2): 288-9.

44. Micheli FE, Pardal MM, Giannaula R, Gatto M, Parera I, Paradiso G, et al. Movement disorders and depression due to flunarizine and cinnarizine. Mov Disord. 1989:4(2):139-46.

45. Mukai E, Makino N, Fujishiro K. Magnetic resonance imaging of parkinsonism. Rinsho Shinkeigaku. 1989:29(6):720-5.

46. Petri $\mathrm{H}$. Extrapyramidal movement disorders following the use of flunarizine. Ned Tijdschr Geneeskd. 1989:133(3):131.

47. Sa PN, Heinisch LM. Parkinson disease induced by flunarizine. Arq Neuropsiquiatr. 1989;47(4):471-3.

48. Centonze V, Magrone D, Vino M, Caporaletti P, Attolini E, Campanale G, et al. Flunarizine in migraine prophylaxis: efficacy and tolerability of $5 \mathrm{mg}$ and $10 \mathrm{mg}$ dose levels. Cephalalgia. 1990;10(1):17-24.

49. Micheli F, Gatto M, Lekhuniec E, Mangone C, Fernandez Pardal M, Pikielny R, et al. Treatment of Tourette's syndrome with calcium antagonists. Clin Neuropharmacol. 1990;13(1):77-83.

50. Senard JM, Colomes M, Rostin M, Clanet M, Montastruc JL. Extrapyramidal syndromes caused by flunarizine. Apropos of 6 cases. Therapie. 1990;45(2): 157-9.

51. Trevisol-Bittencourt P. Flunarizina induzindo parkinsonismo. Arq Cat Med. 1990;19(1):81-4

52. Fontanari JL. Discinesia tardia residual 24 meses após a suspensão do uso da flunarizina. Rev Bras Neurol. 1991;27(3):97-8.

53. Giménez-Roldán S, Mateo D. Cinnarizine-induced parkinsonism. Susceptibility related to aging and essential tremor. Clin Neuropharmacol. 1991;14(2):156-64.

54. Rodríguez García JL, Arechaga US. Flunarizine-induced akathisia. Rev Clin Esp. 1991;188(7):384.

55. Wilder-Smith $\mathrm{CH}$, Schimke J, Osterwalder B, Senn HJ. Cinnarizine for prevention of nausea and vomiting during platin chemotherapy. Acta Oncol. 1991:30(6):731-4.

56. García-Ruiz PJ, García de Yébenes J, Jiménez-Jiménez FJ, Vázquez A, García Urra D, Morales B. Parkinsonism associated with calcium channel blockers: a prospective follow-up study. Clin Neuropharmacol. 1992;15(1):19-26.

57. Morgante L, Rocca WA, Di Rosa AE, De Domenico P, Grigoletto F, Meneghini F, et al. Prevalence of Parkinson's disease and other types of parkinsonism: a door-to-door survey in three Sicilian municipalities. The Sicilian Neuro-Epidemiologic Study (SNES) Group. Neurology. 1992;42(10): 1901-7

58. Negrotti A, Calzetti S, Sasso E. Calcium-entry blockers-induced parkinsonism: possible role of inherited susceptibility. Neurotoxicology. 1992;13(1):261-4.

59. Amancio E, Zymberg S, Peluso C. Parkinsonism and depression induced by flunarizine. Revista Brasileira de Medicina. 1993;50:1316.

60. Anand KS, Thiagarajan A. Flunarizine induced akathisia and depression. Neurol India. 1993;41(4):239.

61. Cunha CA, Bittencourt PRM, Kohlscheen K-L, Mercer LM. Parkinsonisno reversível induzido por cinarizina e flunarizina. Rev méd Paraná. 1993;50(3/ 4):13-6.

62. Curran T, Lang AE. Flunarizine in essential tremor. Clin Neuropharmacol. 1993;16(5):460-3.

63. Galhardo I, Coutinho MO, De Albuquerque ES. Medeiros LdO. Parkinson disease induced by flunarizine: report of a case. Arq Neuropsiquiatr. 1993; 51(4):546-8.

64. Beghi $E$, Monticelli ML, Sessa A, Simone P. The prevalence of parkinsonism in Italy: an epidemiological survey of the disease in general practice. The Italian General Practitioner Study Group (IGPSG). Mov Disord. 1994;9(4):403-8

65. Llau ME, Nguyen L, Senard JM, Rascol O, Montastruc JL. Drug-induced parkinsonian syndromes: a 10-year experience at a regional center of pharmaco-vigilance. Rev Neurol (Paris). 1994;150(11):757-62.

66. Anjaneyulu A, Mohandas S. Flunarizine induced parkinsonism : a report of two cases. Neurol India. 1995;43(4):217-8.
67. Baquero M, Domínguez-Sanz FJ, Burguera JA, Cruz Campos GA, Muñoz R. New forms of parkinsonism in hospice care. Rev Neurol. 1995;23(123):964-8.

68. Brücke T, Wöber C, Podreka I, Wöber-Bingöl C, Asenbaum S, Aull S, et al. D2 receptor blockade by flunarizine and cinnarizine explains extrapyramidal side effects. A SPECT study. J Cereb Blood Flow Metab. 1995;15(3):513-8.

69. Claps A. Parkinson's disease secondary to flunarizine or other drugs. Rev Med Chil. 1995;123(8):1033-6.

70. Handforth A, Mai T, Treiman DM. Rising dose study of safety and tolerance of flunarizine. Eur J Clin Pharmacol. 1995:49(1-2):91-4.

71. Jiménez-Jiménez FJ, Ortí-Pareja M, Ayuso-Peralta L, Gasalla T, CabreraValdivia F, Vaquero A, et al. Drug-induced parkinsonism in a movement disorders unit: a four-year survey. Parkinsonism Relat Disord. 1996;2(3):145-9.

72. Lee SJ, Lee WY. Three cases of flunarizine-induced Parkinsinism. J Korean Neurol Assoc. 1996;14(4):995

73. Martí Massó JF, Poza JJ. Drug-induced or aggravated parkinsonism: clinical signs and the changing pattern of implicated drugs. Neurologia. 1996;11(1): $10-5$

74. Peña MA. Parkinsonismo farmacológico inducido por flunarizina y cinarizina. Rev otorrinolaringol cir cabeza cuello. 1996;56(2):81-3.

75. Morgante L, Di Rosa AE, Savettieri G, Reggio A, Patti F, Salemi G, et al. Druginduced parkinsonism: prevalence, clinical features and follow-up study in three Sicilian communities. J Neurol. 1996;243(3):293-5.

76. Vecchio I, Rampello L, Tornali C, Malaguarnera M, Raffaele R. Flunarizine and essential tremor in the elderly. Arch Gerontol Geriatr. 1996;22(Suppl 1):73-7.

77. Verspeelt J, De Locht P, Amery WK. Postmarketing study of the use of flunarizine in vestibular vertigo and in migraine. Eur J Clin Pharmacol. 1996; 51(1):15-22.

78. Koukoulis A, Herrero JS, Gómez-Alonso J. Blepharospasm induced by flunarizine. J Neurol Neurosurg Psychiatry. 1997;63(3):412-3.

79. Negrotti A, Calzetti S. A long-term follow-up study of cinnarizine- and flunarizine-induced parkinsonism. Mov Disord. 1997;12(1):107-10.

80. Cardoso F, Camargos ST, Silva Júnior GA. Etiology of parkinsonism in a Brazilian movement disorders clinic. Arq Neuropsiquiatr. 1998;56(2):171-5.

81. Errea-Abad JM, Ara-Callizo JR, Aibar-Remón C. Drug-induced parkinsonism. Clinical aspects compared with Parkinson disease. Rev Neurol. 1998;27(155): 35-9.

82. García-Ruiz PJ, Javier Jiménez-Jiménez F, García de Yébenes J. Calcium channel blocker-induced parkinsonism: clinical features and comparisons with Parkinson's disease. Parkinsonism Relat Disord. 1998;4(4):211-4.

83. Martí-Massó JF, Poza JJ. Cinnarizine-induced parkinsonism: ten years later. Mov Disord. 1998;13(3):453-6.

84. Ortí-Pareja M, Jiménez-Jiménez FJ, Vázquez A, Catalán MJ, Zurdo M, Burguera JA, et al. Drug-induced tardive syndromes. Parkinsonism Relat Disord. 1999:5(1-2):59-65.

85. Stucchi-Portocarrero S, Vega-Dienstmaier JM, Saavedra JE, Sagástegui A. Akathisia, parkinsonism and depression induced by cinnarizine: a case report. Rev Neurol. 1999:28(9):876-8.

86. Argote E, Zamora T. Características clínicas y fármacos recibidos por los pacientes con diagnóstico de Parkinson. Colombia Médica. 1999;30(4):16772.

87. Vázquez-Alén P, Pérez-Gilabert Y, Mateo D, Giménez-Roldán S. A study made on a data base of 2,471 patients with Parkinson disease and disorders of movement in Health District 1 of the autonomous region of Madrid. Observed demographic changes over 8 year-period. Rev Neurol. 2000;30(7): 635-40.

88. Schillevoort I, de Boer A, Roos R, Jansen P, Leufkens H. Chapter 4.3 Extrapyramidal syndromes associated with cinnarizine and flunarizine. In: Drug-induced extrapyramidal syndromes. Cologne: Prima Print; 2002.

89. Benito-León J, Bermejo-Pareja F, Rodríguez J, Molina JA, Gabriel R, Morales $J M$, et al. Prevalence of PD and other types of parkinsonism in three elderly populations of central Spain. Mov Disord. 2003;18(3):267-74.

90. Fabiani G, Pastro PC, Froehner C. Parkinsonism and other movement disorders in outpatients in chronic use of cinnarizine and flunarizine. Arq Neuropsiquiatr. 2004;62(3b):784-8.

91. Martí Massó J-F, Martí I, Carrera N, Poza J-J, López de Munain A. Trimetazidine induces parkinsonism, gait disorders and tremor. Therapie. 2005;60(4):419-22

92. Trevisol-Bittencourt P, Pioner L, Tomaselli P, Nahoum R, Tournier M, Collares C, et al. Parkinsonism at a nursing facility. J Neurol Sci. 2005.

93. Alonso-Navarro H, Jiménez-Jiménez FJ. Tardive blepharospasm associated with cinnarizine use. Clin Neuropharmacol. 2006;29(4):187-9. 
94. Barbosa MT, Caramelli P, Maia DP, Cunningham MCQ, Guerra HL, Lima-Costa MF, et al. Parkinsonism and Parkinson's disease in the elderly: a communitybased survey in Brazil (the Bambuí study). Mov Disord. 2006;21 (6):800-8.

95. Turner D, Lurie Y, Finkelstein Y, Schmid T, Gopher A, Kleid D, et al. Pediatric cinnarizine overdose and toxicokinetics. Pediatrics. 2006;117(5):e1067-e9.

96. Benito-León J, Louis ED, Bermejo-Pareja F, Neurological Disorders in Central Spain Study G. Risk of incident Parkinson's disease and parkinsonism in essential tremor: a population based study. J Neurol Neurosurg Psychiatry. 2009;80(4):423-5.

97. Biary N, Al Deeb SM, Bahou Y. Long-term therapy of essential tremor with flunarizine. Eur Neurol. 1995;35(4):217-9.

98. Bisol LW, Brunstein MG, Ottoni GL, Ramos FLP, Borba DL, Daltio CS, et al. Is flunarizine a long-acting oral atypical antipsychotic? A randomized clinical trial versus haloperidol for the treatment of schizophrenia. J Clin Psychiatry. 2008;69(10):1572-9.

99. Diaz-Corrales FJ, Sanz-Viedma S, Garcia-Solis D, Escobar-Delgado T, Mir P. Clinical features and 123I-FP-CIT SPECT imaging in drug-induced parkinsonism and Parkinson's disease. Eur J Nucl Med Mol Imaging. 2010;37(3):556-64.

100. Louter M, Tromp SC. Parkinsonism due to the medication. Ned Tijdschr Geneeskd. 2009;153:A336-A.

101. Ma H-I, Kim J-H, Chu M-K, Oh M-S, Yu K-H, Kim J, et al. Diabetes mellitus and drug-induced parkinsonism: a case-control study. J Neurol Sci. 2009; 284(1-2):140-3.

102. Mattos JP, Nicaretta DH, Rosso ALZ. Progressive supranuclear palsy-like syndrome induced by amiodarone and flunarizine. Arq Neuropsiquiatr. 2009;67(3B):909-10.

103. Munhoz RP, Werneck LC, Teive HAG. The differential diagnoses of parkinsonism: findings from a cohort of 1528 patients and a 10 years comparison in tertiary movement disorders clinics. Clin Neurol Neurosurg. 2010;112(5):431-5.

104. Bondon-Guitton E, Perez-Lloret S, Bagheri H, Brefel C, Rascol O, Montastruc J-L. Drug-induced parkinsonism: a review of 17 years' experience in a regional pharmacovigilance center in France. Mov Disord. 2011;26(12):2226-31.

105. Kizilay F, Ekmekci B, Gungor H, Uysal H, Oh SJ. Flunarizine-induced fasciculation-myokymia. J Clin Neuromuscul Dis. 2011;12(4):246-7.

106. Masmoudi K, Masson H, Gras V, Andréjak M. Extrapyramidal adverse drug reactions associated with trimetazidine: a series of 21 cases. Fundam Clin Pharmacol. 2012;26(2):198-203.

107. Arias AC, Reyes ED, Fernández JCD, Pérez RC, González LRA, Nogueira GC, et al. Depresión Mayor inducida por flunarizina. Associacion colombiana de Psiquiatria. 2012;07:11-2.

108. Foubert-Samier A, Helmer C, Perez F, Le Goff M, Auriacombe S, Elbaz A, et al. Past exposure to neuroleptic drugs and risk of Parkinson disease in an elderly cohort. Neurology. 2012;79(15):1615-21.

109. Pioner LM. Relato de parkinsonismo induzido por cinarizina: reflexões sobre uma iatrogenia. Revista Espaço Acadêmico. 2012;12(136):122-9.

110. Kim JS, Youn J, Shin H, Cho JW. Nonmotor symptoms in drug-induced parkinsonism and drug-naïve Parkinson disease. Can J Neurol Sci. 2013;40(1):36-41.

111. DR G, LE C, EL R, AL M. Flunarizine-induced parkinsonism. Brazilian Journal of Surgery and Clinical Research. 2014;7(2):25-7.

112. Miguel R, Correia ASA, Bugalho P. latrogenic parkinsonism: the role of flunarizine and cinnarizine. J Park Dis. 2014;4(4):645-9.

113. Otero S. Flunarizine and neuro-psychiatric symptoms in children and adolescents. J Pediatr Neurol. 2006;4(4):271-3.

114. Krishnan Vengadaragava C, Raman K. A unique case of flunarizine induced extrapyramidal syndrome and depression. PTB Reports. 2016;2(2).

115. Lin H-L, Lin H-C, Tseng Y-F, Chen S-C, Hsu C-Y. Risk of parkinsonism induced by flunarizine or cinnarizine: a population-based study. Eur J Clin Pharmacol. 2017;73(3):365-71.

116. Munhoz RP, Bertucci Filho D, Teive HAG. Not all drug-induced parkinsonism are the same: the effect of drug class on motor phenotype. Neurol Sci. 2017;38(2):319-24.

117. Nisticò R, Fratto A, Vescio B, Arabia G, Sciacca G, Morelli M, et al. Tremor pattern differentiates drug-induced resting tremor from Parkinson disease. Parkinsonism Relat Disord. 2016;25:100-3.

118. Sung YH, Noh Y, Lee J, Kim EY. Drug-induced parkinsonism versus idiopathic Parkinson disease: itility of nigrosome 1 with 3-T imaging. Radiology. 2016;279(3):849-58.

119. Jhang K-M, Huang J-Y, Nfor ON, Tung Y-C, Ku W-Y, Lee C-T, et al. Erratum to: Extrapyramidal symptoms after exposure to calcium channel blockerflunarizine or cinnarizine. Eur J Clin Pharmacol. 2017;73(7):917.
120. Lopez-Castellanos JR, Lopez-Contreras J, editors. Myoclonus and tremor induced by flunarizine and cinnarizine: a report of four cases in El Salvador. Movement Disorders; 2017

121. Mathews SR, Badyal DK, Varghese A. Acute dystonia induced by prochlorperazine and cinnarizine. J Rational Pharmacother Res. 2017;3:53-4.

122. Yang YW, Hsieh TF, Li Cl, Liu CS, Lin WY, Chiang JH, et al. Increased risk of Parkinson disease with diabetes mellitus in a population-based study. Medicine. 2017;96(3):e5921.

123. Karsan N, Palethorpe D, Rattanawong W, Marin JC, Bhola R, Goadsby PJ. Flunarizine in migraine-related headache prevention: results from 200 patients treated in the UK. Eur J Neurol. 2018;25(6):811-7.

124. Liang C-Y, Yeh Y-C, Lee C-J, Chen Y-Y. Flunarizine and the risk of parkinsonism in a newly diagnosed type 2 diabetic population in Taiwan: a nested case-control study. J Clin Neurosci. 2018;50:281-6.

125. Byun JH, Cho H, Kim YJ, Kim JS, Baik JS, Jang S, et al. Trends in the prevalence of drug-induced parkinsonism in Korea. Yonsei Med J. 2019; 60(8):760-7.

126. Gallop F, Fosi T, Prabhakar P, Aylett SE. Flunarizine for headache prophylaxis in children with Sturge-Weber syndrome. Pediatr Neurol. 2019;93:27-33.

127. Jhang K-M, Huang J-Y, Nfor ON, Tung Y-C, Ku W-Y, Jan C-F, et al. Flunarizine related movement disorders: a nationwide population-based study. Sci Rep. 2019:9(1):1705.

128. Kim S, Cheon S-M, Suh HS. Association between drug exposure and occurrence of parkinsonism in Korea: a population-based case-control study. Ann Pharmacother. 2019;53(11):1102-10.

129. Lin W, Lin C-L, Hsu CY, Wei C-Y. Flunarizine induced parkinsonism in migraine group: a nationwide population-based study. Front Pharmacol. 2019;10:1495.

130. Teive HAG, Munhoz RP, Ferraz HB. Flunarizine and cinnarizine-induced parkinsonism: 25 years of de Melo-Souza's syndrome. Arq Neuropsiquiatr. 2009;67(3B):957.

131. Teive HAG, Troiano AR, Germiniani FMB, Werneck LC. Flunarizine and cinnarizine-induced parkinsonism: a historical and clinical analysis. Parkinsonism Relat Disord. 2004;10(4):243-5.

132. Brugger F, Bhatia KP, Besag FM. Valproate-associated parkinsonism: a critical review of the literature. CNS drugs. 2016;30(6):527-40.

133. Gerber PE, Lynd LD. Selective serotonin-reuptake inhibitor-induced movement disorders. Ann Pharmacother. 1998;32(6):692-8.

134. Lepping P, Delieu J, Mellor R, Williams JHH, Hudson PR, Hunter-Lavin C. Antipsychotic medication and oxidative cell stress: a systematic review. J Clin Psychiatry. 2011;72(3):273-85.

135. Blandina P, Munari L, Provensi G, Passani MB. Histamine neurons in the tuberomamillary nucleus: a whole center or distinct subpopulations? Front Syst Neurosci. 2012;6:33.

136. Rupniak NM, Jenner $P$, Marsden CD. Acute dystonia induced by neuroleptic drugs. Psychopharmacology. 1986;88(4):403-19.

137. Jimenez-Jimenez FJ, Puertas I, de Toledo-Heras M. Drug-induced myoclonus. CNS drugs. 2004;18(2):93-104.

\section{Publisher's Note}

Springer Nature remains neutral with regard to jurisdictional claims in published maps and institutional affiliations.

\section{Submit your manuscript to a SpringerOpen ${ }^{\circ}$ journal and benefit from:}

- Convenient online submission

- Rigorous peer review

- Open access: articles freely available online

- High visibility within the field

- Retaining the copyright to your article

Submit your next manuscript at $>$ springeropen.com 